\title{
Probing Electrochemistry at Nanoscale: In Situ TEM and STM Characterizations of Conducting Filaments in Memristive Devices
}

\author{
Yuchao Yang ${ }^{*}$, Yasuo Takahashi ${ }^{2 *}$, Atsushi Tsurumaki-Fukuchi ${ }^{2}$, Masashi Arita $^{2 *}$, M. Moors ${ }^{3 *}$, M. \\ Buckwell $^{4}$, A. Mehonic ${ }^{4}$, and A. J. Kenyon ${ }^{4 *}$ \\ ${ }^{1}$ Key Laboratory of Microelectronic Devices and Circuits (MOE), Institute of Microelectronics, \\ Peking University, Beijing 100871, China
}

${ }^{2}$ Graduate School of Information Science and Technology, Hokkaido University, Sapporo, 060-0814, Japan

${ }^{3}$ Peter Grünberg Institute, Forschungszentrum Jülich, Wilhelm-Johnen-Straße, D-52425 Jülich, Germany

${ }^{4}$ Department of Electronic \& Electrical Engineering, University College London, Torrington Place, London WC1E 7JE, United Kingdom

*Correspondence to: yuchaoyang@pku.edu.cn $\quad$ (Y.Y.), y-taka@ist.hokudai.ac.jp (Y.T.), arita@nano.ist.hokudai.ac.jp (M.A.),m.moors@fz-juelich.de (M.M.), a.kenyon@ucl.ac.uk (A.J.K.)

\begin{abstract}
Memristors or memristive devices are two-terminal nanoionic systems whose resistance switching effects are induced by ion transport and redox reactions in confined spaces down to nanometre or even atomic scales. Understanding such localized and inhomogeneous electrochemical processes is a challenging but crucial task for continued applications of memristors in nonvolatile memory, reconfigurable logic, and brain inspired computing. Here we give a survey for two of the most powerful technologies that are capable of probing the resistance switching mechanisms at the nanoscale - transmission electron microscopy, especially in situ, and scanning tunneling microscopy, for memristive systems based on both electrochemical metallization and valence changes. These studies yield rich information about the size, morphology, composition, chemical state and


growth/dissolution dynamics of conducting filaments and even individual metal nanoclusters, and have greatly facilitated the understanding of the underlying mechanisms of memristive switching. Further characterization of cyclic operations leads to additional insights into the degradation in performance, which is important for continued device optimization towards practical applications.

Keywords Resistive random access memory, Conducting filament, Transmission electron microscopy, Scanning tunneling microscopy, In situ, Electrochemical reactions

Resistive Random Access Memories (ReRAMs), or memristors [1], have tremendous potential for applications in nonvolatile embedded or storage class memories [2, 3], as well as in-memory logic that overcomes the von Neumann bottleneck [4]. They are also envisaged to form the basis of power-efficient neuromorphic systems by implementing plasticity similar to that of biological synapses [5]. Consequently, extensive efforts have been devoted to research into this novel class of devices, including studies of switching materials, device structures, scalability, integration, and so on. In particular, it is very important to understand the fundamental operation mechanisms of ReRAM in order to guide device-related studies. It is well known that the resistance switching effects in memristors are usually modulated by ionic transport and subsequent conducting filament formation/dissolution processes occurring at dimensions around $10 \mathrm{~nm}$, and even down to atomic scales [6-8], hence elucidating the switching mechanisms requires advanced characterization techniques capable of resolving the nanoscale switching regions formed randomly in the devices. Here we give a survey of two of the most important technologies in understanding the switching mechanisms of ReRAM - transmission electron microscopy (TEM), especially when applied in situ, and scanning tunneling microscopy (STM). We will discuss the principles and designs behind these techniques, and show how they have furthered the understanding of the nature and dynamic evolution of conductive filaments. Finally, the remaining challenges in the understanding of memristive mechanisms will be briefly discussed. 


\section{In situ TEM characterization of metal filament-based ReRAM}

\subsection{Sample structures enabling in situ TEM observations}

TEM is a widely adopted technique in studies of ReRAM. It can achieve direct visualization of the nanoscale localized filaments that are responsible for resistance switching. A number of studies have been performed on both cation migration-based conductive bridge random access memories (CBRAMs) and anion migration-based valence change memories (VCMs). TEM is a microscopic imaging technique in which a beam of electrons is transmitted through an ultra-thin specimen (thickness $<100 \mathrm{~nm}$ ) and an image is subsequently formed from the interaction between the electrons and the sample. Due to the small de Broglie wavelength of electrons, and especially thanks to recent technological advancements such as the introduction of spherical aberration correctors, state-of-the-art TEM instruments have reached unparalleled resolutions beyond the angstrom scale, making TEM one of the most powerful technologies to resolve the structural details of conducting filaments [9-12]. In addition, analytical studies of the filaments can be realized utilizing complementary techniques in TEM, such as energy dispersive X-ray spectroscopy (EDS or EDX), electron energy loss spectroscopy (EELS), and so on, providing further information about the composition and chemical states of filaments. Besides static characterization, in situ TEM observation allows simultaneous monitoring of both electrical switching behavior and real-time evolution of microstructure. Such in situ TEM studies have been widely performed to reveal the filament growth and dissolution dynamics of different ReRAM devices, and have greatly facilitated the understanding of detailed electrochemical processes.

Various sample structures have been carefully designed in order to meet the specific challenges of in situ TEM observations of filament dynamics. In general, the samples should represent the ReRAM devices studied in terms of essential attributes or structures, so that the findings acquired from the model system can be applicable to ReRAM developments. The samples should also be thin enough so that any meaningful structural changes during switching can be visualized by the transmitted electron beam, while they should not lose their programmability at this thickness and inside the TEM chamber. Figure 1 summarizes typical structures that have been adopted in existing studies. A crude classification of existing TEM sample structures divides them into two different configurations: vertical and planar. Figures 1a-c show in situ vertical TEM structures, which better 
resemble the structure of ReRAM cells. For instance, Fig. 1a shows a vertical setup in which a switching dielectric-encapsulated tungsten probe is contacted with a silver wire inside the TEM column [9, 10], forming a typical CBRAM structure of active electrode/dielectric/inert electrode. This facile, nondestructive design allows thin and clean TEM samples to be fabricated that are directly ready for in situ programming and imaging, refraining from contamination and damage that is usually inevitable in traditional sample preparation. In situ vertical TEM samples can also be prepared by focused ion beam (FIB) cutting [13] or traditional manual grinding followed by ion milling [14], as shown in Figs. 1b and 1c, respectively. In situ TEM studies have also been performed on samples in planar configurations [9], as shown in Fig. 1d. Such samples are usually fabricated on ultra-thin $\mathrm{SiN}_{\mathrm{x}}$ membranes, and hence the samples can be directly subjected to TEM imaging without going through any destructive thinning process. The lateral layout renders a clear sight of the switching region and allows one to directly observe any structural changes to the device down to the smallest details. This structure can be further modified by adopting a fabrication order of bottom electrode, electrolyte (switching dielectric), and top electrode, forming a slant structure as shown Fig. 1e, which partly resembles the features of vertical structures [15]. In order to further understand the dynamics in CBRAM from a more fundamental point of view, special attention should be paid to the dynamics of individual metal nanoclusters as well, in addition to that of whole filaments. Figure 1f shows a dedicated sample structure for in situ TEM observations of metal clusters. The details will be discussed in subsequent sections.

(a)

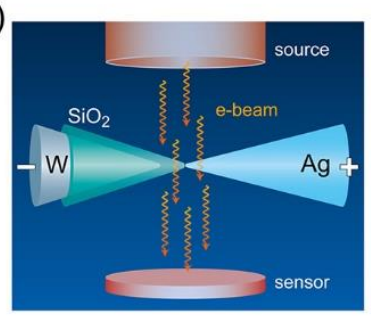

(d)

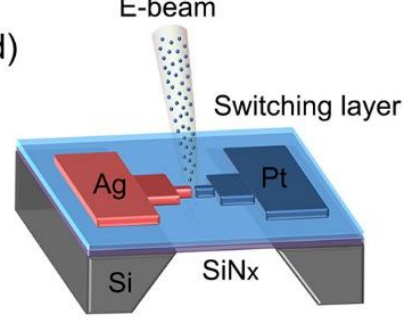

(b)

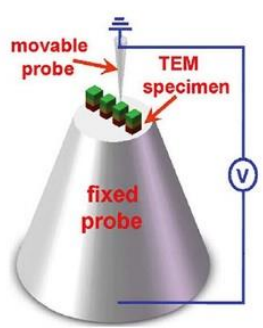

(e)

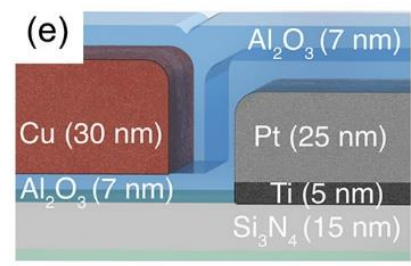

(c)
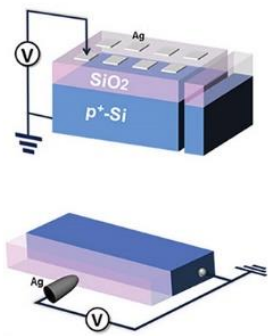

(f)

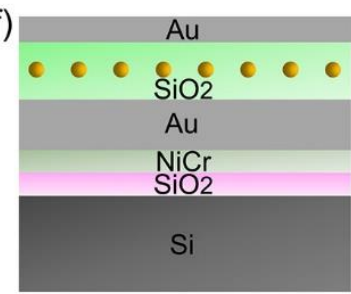

Fig. 1 Schematic illustrations of sample structures for in situ TEM observations. (a) Vertical structure consisting of a 
switching dielectric-encapsulated W probe in contact with a Ag wire. Reprinted from [10] with permission. (b) In situ TEM experimental setup based on cross sections prepared by FIB. Reprinted from [13] with permission. Copyright Wiley-VCH Verlag GmbH \& Co. KGaA. (c) Schematic of ex situ (top panel) and in situ (bottom panel) experimental setups based on cross sections prepared by manual grinding and ion milling. Reprinted from [14] with permission. Copyright Wiley-VCH Verlag GmbH \& Co. KGaA. (d) Schematic of planar device configuration on SiN $\mathrm{N}_{\mathrm{x}}$ membrane. Reprinted from [16] with permission. (e) Planar device configuration with a slant structure. Reprinted from [15] with permission. (f) Schematic of the samples consisting of Ag nanoclusters embedded in $\mathrm{SiO}_{2}$ for in situ TEM observation. Reprinted from [10] with permission

\subsection{Classification of dynamic metal filament growth modes}

Table 1 summarizes existing studies reporting direct observation of conduction channels in CBRAM devices. Studies employing very similar sample structures but based on scanning electron microscopy (SEM) observations were also included for the sake of completeness. Firstly, these studies provide unambiguous evidence for the formation of conducting filaments in ReRAM, hence validating the filamentary theory. In addition, more in-depth understanding was acquired of the composition, crystalline structure and chemical state of the filaments, and even their growth dynamics. In agreement with previous theoretical assumptions, the conduction channels formed in a majority of CBRAM devices were found to be composed of $\mathrm{Ag}$ or $\mathrm{Cu}$ metal filaments, as shown by high-resolution TEM (HRTEM) or electron diffraction analysis [9, 13, 17], although a few reports have also suggested that phase transitions may serve as an alternative cause for the switching in some chalcogenide-based solid electrolytes, such as $\mathrm{Ag}_{2} \mathrm{~S}$ [18] and $\mathrm{Ag}-\mathrm{Ge}-\mathrm{Se}$ [19].

Table 1 Summary of direct observations of metal filament growth and cluster movements in different systems. For an explanation of growth modes, see Fig. 2.

\begin{tabular}{cccccc}
\hline $\begin{array}{c}\text { Configu- } \\
\text { ration }\end{array}$ & Device structure & $\begin{array}{c}\text { Growth } \\
\text { mode }\end{array}$ & Approach & $\begin{array}{c}\text { Research } \\
\text { object }\end{array}$ & Ref. \\
\hline \multirow{4}{*}{ Vertical } & $\mathrm{Ag} / \mathrm{Ag}_{2} \mathrm{~S} / \mathrm{W}$ & - & in situ $\mathrm{TEM}$ & Filament & {$[18]$} \\
& $\mathrm{Cu} / \mathrm{Cu}-\mathrm{GeTe} / \mathrm{Pt}-\mathrm{Ir}$ & $\mathrm{a}$ & in situ $\mathrm{TEM}$ & Filament & {$[20]$} \\
& $\mathrm{Pt}-\mathrm{Ir} / \mathrm{Cu}-\mathrm{GeS} / \mathrm{Pt}-\mathrm{Ir}$ & $\mathrm{a}$ & in situ $\mathrm{TEM}$ & Filament & {$[17]$} \\
$\mathrm{Cu} / \mathrm{Al}_{2} \mathrm{O}_{3} / \mathrm{Pt}$ & $\mathrm{a}$ & in situ $\mathrm{TEM}$ & Filament & {$[15]$} \\
& $\mathrm{Ag}\left(\mathrm{Cu} / \mathrm{ZrO}_{2} / \mathrm{Pt}\right.$ & $\mathrm{b}$ & in situ $\mathrm{TEM}$ & Filament & {$[13]$} \\
\hline
\end{tabular}




\begin{tabular}{|c|c|c|c|c|c|}
\hline & $\mathrm{Ag} / \mathrm{a}-\mathrm{Si}(\mathrm{PECVD}) / \mathrm{Pt}$ & $\mathrm{b}$ & in situ TEM & Filament & [9] \\
\hline & $\mathrm{Ni} / \mathrm{ZrO}_{2} / \mathrm{Pt}$ & - & in situ TEM & Filament & [21] \\
\hline & $\mathrm{Ag} / \mathrm{SiO}_{2}(\mathrm{PECVD}) / \mathrm{p}^{+}-\mathrm{Si}$ & $\mathrm{b}$ & in situ TEM & Filament & {$[14]$} \\
\hline & $\mathrm{Ag} / \mathrm{SiO}_{2}$ (evaporated)/Pt & $\mathrm{b}$ & in situ TEM & Filament & [10] \\
\hline & $\mathrm{Cu} / \mathrm{WO}_{\mathrm{x}} / \mathrm{TiN}$ & - & in situ TEM & Filament & [12] \\
\hline & $\mathrm{Au} / \mathrm{SiO}_{2}(\mathrm{PECVD}) / \mathrm{Ag}$ nanoclusters $/ \mathrm{SiO}_{2} / \mathrm{p}^{+}-\mathrm{Si}$ & $\mathrm{b}$ & in situ TEM & Cluster & [14] \\
\hline & $\mathrm{Au} / \mathrm{SiO}_{2}$ (evaporated) $/ \mathrm{Ag}$ nanoclusters $/ \mathrm{SiO}_{2} / \mathrm{Au}$ & $\mathrm{b}$ & in situ TEM & Cluster & {$[10]$} \\
\hline & $\mathrm{Au} / \mathrm{SiO}_{2}($ evaporated $) / \mathrm{Cu}$ nanoclusters $/ \mathrm{SiO}_{2} / \mathrm{Au}$ & $\mathrm{b}$ & in situ TEM & Cluster & {$[10]$} \\
\hline & $\mathrm{Au} / \mathrm{SiO}_{2}$ (evaporated)/Ni nanoclusters $/ \mathrm{SiO}_{2} / \mathrm{Au}$ & $\mathrm{c}$ & in situ TEM & Cluster & [10] \\
\hline & $\mathrm{Au} / \mathrm{SiO}_{2}$ (evaporated)/Pt nanoclusters $/ \mathrm{SiO}_{2} / \mathrm{Au}$ & $\mathrm{b}$ & in situ TEM & Cluster & {$[10]$} \\
\hline & $\mathrm{Cu} / \mathrm{Ta}_{2} \mathrm{O}_{5} / \mathrm{Pt}$ & - & ex situ TEM & Filament & {$[22]$} \\
\hline & $\mathrm{Ag} / \mathrm{ZnO}: \mathrm{Mn} / \mathrm{Pt}$ & - & ex situ TEM & Filament & [23] \\
\hline & $\mathrm{Ag} / \mathrm{Ag}_{30} \mathrm{Ge}_{17} \mathrm{Se}_{53} / \mathrm{Pt}$ & - & ex situ TEM & Percolation & [19] \\
\hline \multirow{9}{*}{ Planar } & $\mathrm{Ag} / \mathrm{H}_{2} \mathrm{O} / \mathrm{Pt}$ & $\mathrm{a}$ & SEM & Filament & {$[24]$} \\
\hline & $\mathrm{Ag} / \mathrm{SiO}_{2}$ (sputtered) $/ \mathrm{Pt}$ & d & ex situ TEM & Filament & [9] \\
\hline & $\mathrm{Ag} / \mathrm{Al}_{2} \mathrm{O}_{3} / \mathrm{Pt}$ & $\mathrm{b}$ & SEM & Filament & [9] \\
\hline & $\mathrm{Ag} / \mathrm{Ag}-\mathrm{PEO} / \mathrm{Pt}$ & d & SEM & Filament & {$[25]$} \\
\hline & $\mathrm{Ag} / \mathrm{PEO} / \mathrm{Pt}$ & $\mathrm{b}$ & SEM & Filament & {$[25]$} \\
\hline & $\mathrm{Ag} / \mathrm{a}-\mathrm{Si}(\mathrm{PECVD}) / \mathrm{Pt}$ & $\mathrm{b}$ & SEM & Filament & [9] \\
\hline & $\mathrm{Ag} / \mathrm{TiO}_{2} / \mathrm{Pt}$ & - & SEM & Filament & {$[26]$} \\
\hline & $\mathrm{Pd} / \mathrm{SiO}_{\mathrm{x}}($ sputtered $) / \mathrm{Pd}$ & $\mathrm{b}$ & ex situ TEM & Filament & [27] \\
\hline & Ag/PEDOT:PSS/Pt & $\mathrm{b}$ & SEM & Filament & [28] \\
\hline
\end{tabular}

“-" implies the growth mode cannot be determined based on the reported results.

In contrast to the highly consistent results on filament composition, recent in situ TEM observations performed on different device systems, as listed in Table 1, have exhibited diverse results on the switching dynamics, including the filament growth direction (initiating from the active electrode $[9,10,13,14]$ or from the inert electrode $[15,20,24]$ ), filament geometry (forward cone/dendrite $[9,10,25]$, backward cone/dendrite [20], or cylindrical filament [13, 22, 23]), and filament morphology (continuous metal wire [13, 15, 17, 22-24] or a chain of individual metal clusters $[9,26])$. These findings have greatly enriched the understanding of the electrochemical processes occurring in ReRAM, as well as the resultant filament growth dynamics. Notably, a careful examination of the growth behaviors listed in Table 1 can divide them into 4 regimes (depicted in Fig. 2), except for a few studies where full details about the growth dynamics cannot be determined yet from existing information. These different filament growth modes in different device systems can be ascribed to the variations in kinetic parameters governing the growth process, with ion mobility $(\mu)$ and redox reaction rate $(\Gamma)$ being two of the most important kinetic parameters. Below we will 
discuss these different growth modes, together with experimental observations from in situ TEM studies.

(a)

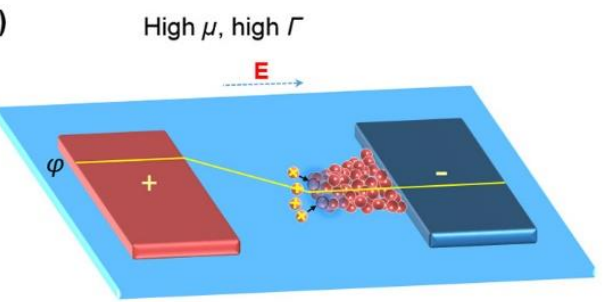

(c)

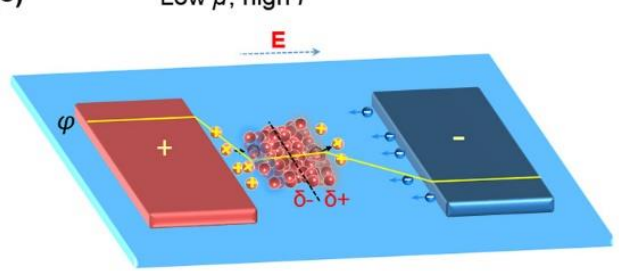

(b)

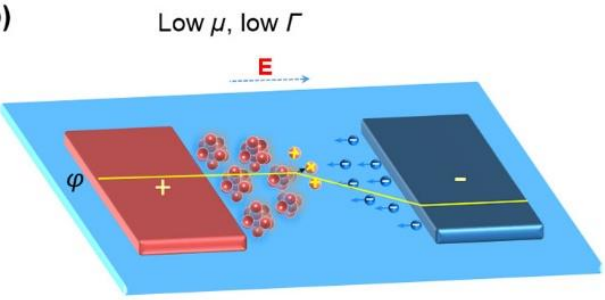

(d)

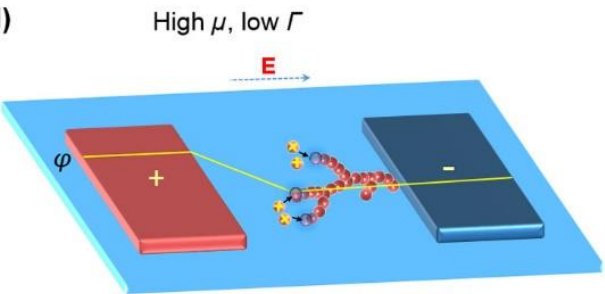

Fig. 2 Qualitative model showing different filament growth modes governed by kinetic parameters. (a) growth mode a: both $\mu$ and $\Gamma$ are high, resulting in filament growth from the inert electrode and an inverted cone shape. (b) growth mode b: both $\mu$ and $\Gamma$ are low, resulting in filament growth from the active electrode with discrete nanoclusters and a forward cone shape. (c) growth mode c: $\mu$ is low but $\Gamma$ is high, resulting in filament nucleation inside the dielectric and backward growth. (d) growth mode $\mathrm{d}: \mu$ is high but $\Gamma$ is low, resulting in filament growth from the inert electrode and a dendrite structure. Reprinted from [10] with permission

\subsubsection{High ion mobility, high redox rate case}

When the ion mobility in the switching dielectric is high and homogeneous, it is expected that the metal ions can reach the inert electrode quickly, and thus the growth should initiate from there. Meanwhile, a high redox reaction rate results in the reduction of large numbers of ions at the inert electrode interface, leading to inverted cone-shaped filaments, as schematically shown in Fig. 2a. In reality, the properties of high $\mu$ and high $\Gamma$ are usually found in conventional solid electrolytes [2, 29], and the growth dynamics depicted in Fig. 2a have been directly verified by in situ TEM and SEM observations in $\mathrm{Cu} / \mathrm{Cu}-\mathrm{GeTe} / \mathrm{Pt}-\mathrm{Ir}$ [20], $\mathrm{Pt}-\mathrm{Ir} / \mathrm{Cu}-\mathrm{GeS} / \mathrm{Pt}-\mathrm{Ir}$ [17], $\mathrm{Cu} / \mathrm{Al}_{2} \mathrm{O}_{3} / \mathrm{Pt}$ [15], and $\mathrm{Ag} / \mathrm{H}_{2} \mathrm{O} / \mathrm{Pt}$ devices [24] (Fig. 3). Note, that the irregular filament geometry in the Pt-Ir/Cu-GeS/Pt-Ir device shown in Fig. 3d is likely a result of the asymmetric electrode shape (one electrode is a tip and the other is planar) rather than an intrinsic effect. The dissolution process in this mode is considered to 
be a reverse process of the filament growth, where the formed filament dissolves at its thinnest part, namely, the active electrode interface. This dissolution process has also been experimentally verified by in situ TEM observations [20].
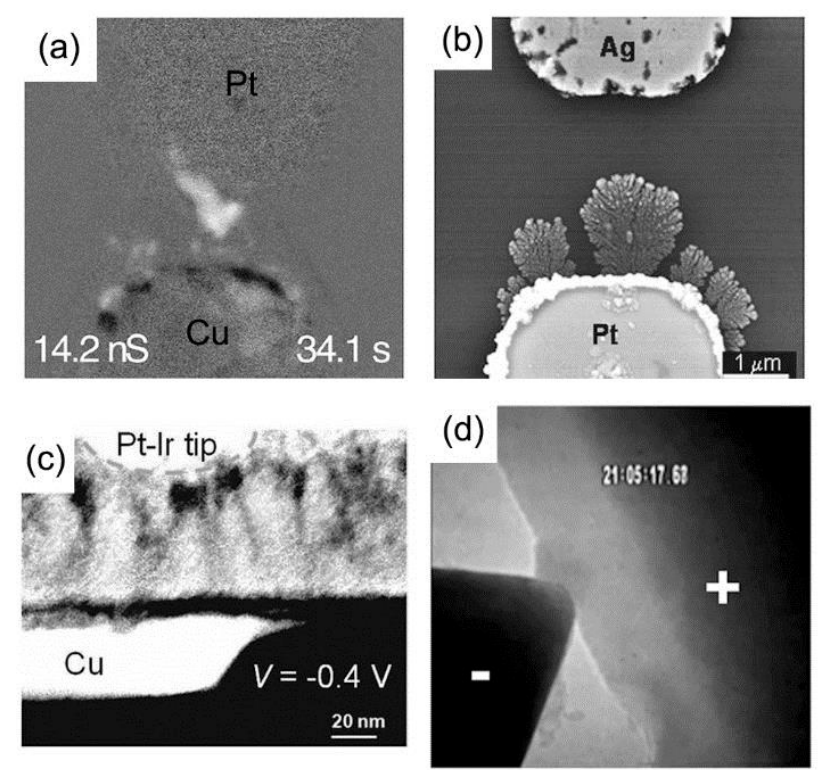

Fig. 3 Direct observations of filament growth dynamics in (a) $\mathrm{Cu} / \mathrm{Al}_{2} \mathrm{O}_{3} / \mathrm{Pt}$, Reprinted from [15] with permission, (b) $\mathrm{Ag} / \mathrm{H}_{2} \mathrm{O} / \mathrm{Pt}$, Reprinted from [24] with permission, (c) $\mathrm{Cu} / \mathrm{Cu}-\mathrm{GeTe} / \mathrm{Pt}-\mathrm{Ir}$, Reprinted from [20] with permission, Copyright Wiley-VCH Verlag GmbH \& Co. KGaA, (d) Pt-Ir/Cu-GeS/Pt-Ir devices. Reprinted from [17] with permission

\subsubsection{Low ion mobility, low redox rate case}

In the other extreme case of low $\mu$ and low $\Gamma$ (case b, Fig. 2b), the ions may only be able to travel a short distance from the active electrode before achieving the critical nucleation conditions inside the dielectric, leading to filament growth from the active electrode to the inert electrode. Figures $4 \mathrm{a}-\mathrm{d}$ present a sequence of TEM images from a real-time, in situ TEM movie recorded in the forming process of $\mathrm{Ag} / \mathrm{a}-\mathrm{Si} / \mathrm{Pt}$ devices, which unambiguously show this growth process. Such a growth mode significantly departs from the filament dynamics in chalcogenide-based solid electrolytes, and has also been experimentally verified in a number of other CBRAM systems, including $\mathrm{Ag} / \mathrm{ZrO}_{2} / \mathrm{Pt}$ [13], Ag/evaporated-SiO $2 / \mathrm{Pt}$ [10], and $\mathrm{Ag} / \mathrm{PECVD}$ (plasma enhanced chemical vapor deposition)- $\mathrm{SiO}_{2} / \mathrm{Pt}$ devices [14], as shown in Figs. 4e, 4f, and 4g, respectively. Importantly, the filament is usually not a continuous metal wire, but is instead composed of discrete metal 
nanoclusters, due to the low redox reaction rates, as observed in $\mathrm{Ag} / \mathrm{a}-\mathrm{Si} / \mathrm{Pt}$ [9], $\mathrm{Ag} /$ evaporated-SiO $2 / \mathrm{Pt}[10]$, and $\mathrm{Ag} / \mathrm{PECVD}-\mathrm{SiO}_{2} / \mathrm{Pt}$ devices [14] (Figs. 4a-d, 4f and 4g). In this case, the high conductance in the on state is presumably a result of electrons tunneling through the nanogaps between neighboring metal clusters. It can be noticed that this growth mode typically happens in non-conventional electrolytes, such as oxides, with dramatically different material properties from chalcogenides. Also, since the kinetic parameters are closely correlated with the microstructure of the dielectrics - especially the type, number and distribution of defects - even materials with the same composition but prepared by different deposition techniques can have significantly different behaviors. For example, $\mathrm{SiO}_{2}$ films deposited by electron beam evaporation and PECVD have relatively dense microstructures and consequently rather low $\mu$, and the filament growth falls into mode b. However, $\mathrm{SiO}_{2}$ films prepared by sputtering have relatively open microstructures containing a large number of defects. The ion mobility in the sputtered $\mathrm{SiO}_{2}$ films is thus enhanced, resulting in filament growth initiated from the inert electrode, following mode $d$, which we discuss below [9].
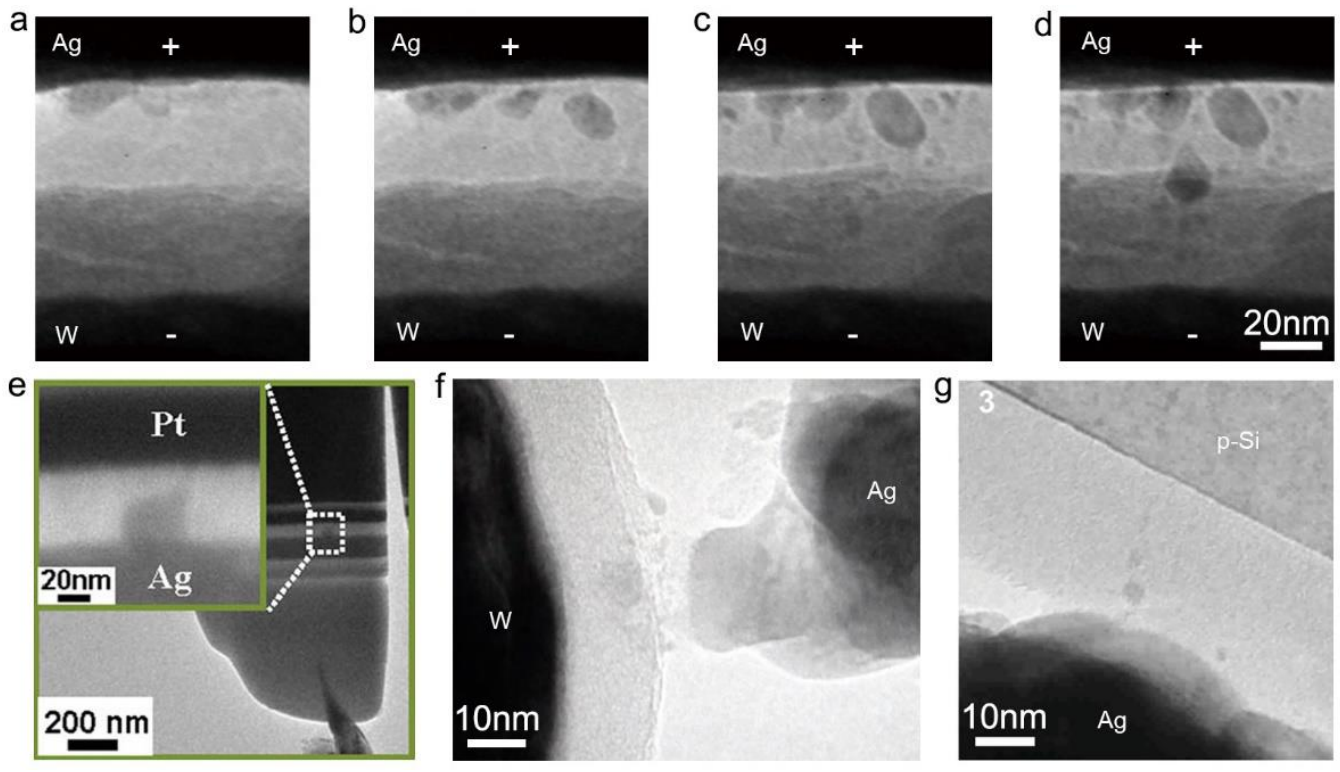

Fig. 4 (a-d) In situ TEM observation of filament growth dynamics in vertical Ag/a-Si/W memories. Scale bar, $20 \mathrm{~nm}$. Reprinted from [9] with permission. (e) Partially formed filament in an $\mathrm{Ag} / \mathrm{ZrO}_{2} / \mathrm{Pt}$ device. Reprinted from [13] with permission. Copyright Wiley-VCH Verlag GmbH \& Co. KGaA. (f) Partially formed filament in an $\mathrm{Ag} /$ evaporated-SiO$/ 2 / \mathrm{Pt}$ device. Reprinted from [10] with permission. (g) Partially formed filament in an $\mathrm{Ag} / \mathrm{PECVD}-\mathrm{SiO}_{2} / \mathrm{Pt}$ device. Reprinted from [14] with permission, Copyright Wiley-VCH Verlag GmbH \& Co. KGaA 


\subsubsection{Low ion mobility, high redox rate case}

In the case of low $\mu$ and high $\Gamma$ (case c, Fig. 2c), the nucleation of filaments should also take place inside the dielectric, similar to mode $b$. The difference lies in the high $\Gamma$ in this case, which can result in persistent reduction of metal ions at the nucleation site, leading to backward filament growth from the nucleation site to the active electrode. Indeed, this type of growth was observed by in situ TEM imaging in the case of Ni filament growth in evaporated $\mathrm{SiO}_{2}$ films, as shown in Fig. 5 [10]. Here, the low ion mobility can be ascribed to the dense microstructure of the evaporated film, and the high $\Gamma$ was experimentally realized by the introduction of a large Ni source serving as the ion supply.
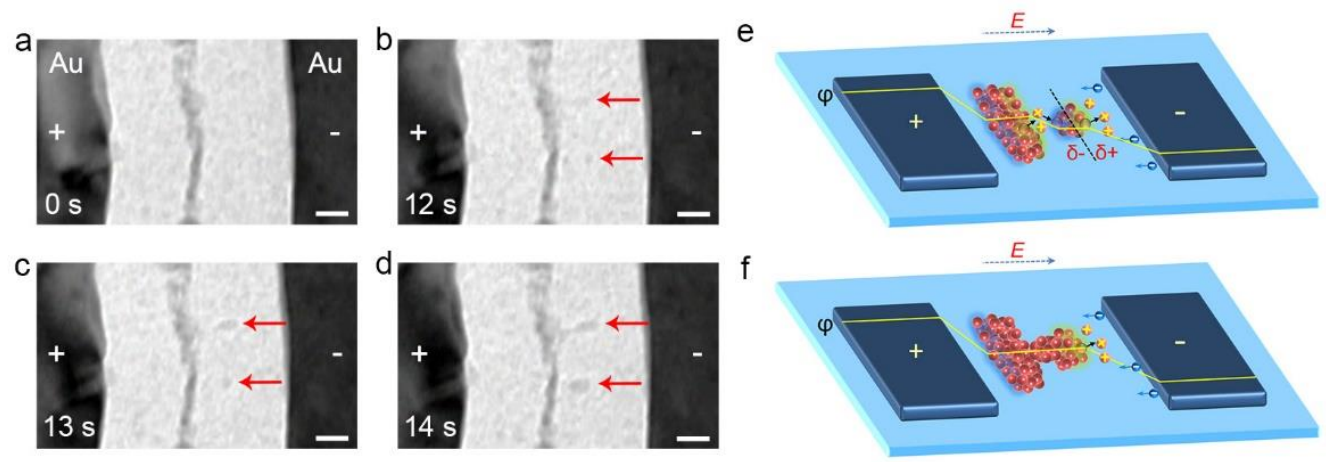

Fig. 5 Dynamic growth and migration of $\mathrm{Ni}$ in $\mathrm{SiO}_{2}$. (a-d) In situ TEM images showing the evolution of Ni nanoclusters. Scale bar, $10 \mathrm{~nm}$. (e) Schematic of cluster nucleation from a large embedded metal source. (f) Schematic of the backward growth from the nucleus due to a high redox reaction rate, forming a solid connection with the source. Reprinted from [10] with permission

\subsubsection{High ion mobility, low redox rate case}

In the last case of high $\mu$ and low $\Gamma$ (case d, Fig. 2d), the nucleation of filaments is expected to occur at the inert electrode due to the fast movement of ions. However, in contrast to mode a, which has a large ion supply, the limited redox reaction rate in this case determines that ion reduction will preferably take place at the edges of existing filaments with high field strengths, leading to branched filament growth towards the active electrode. This phenomenon was directly observed in the case of 
Ag filament growth in sputtered $\mathrm{SiO}_{2}$ films, as shown in Fig. 6a, where the high $\mathrm{Ag}$ ion mobility was a result of the defective microstructure of sputtered $\mathrm{SiO}_{2}$.

A consequence of such a dendrite structure is that the thinnest part of the filament appears at the inert electrode interface, forming a region with enhanced electric field. As a result, subsequent filament dissolution also occurs to this region, as demonstrated in Fig. $6 \mathrm{~b}$ and its inset [9]. Recently, this filament growth mode has also been observed in $\mathrm{Ag} / \mathrm{Ag}-\mathrm{PEO} / \mathrm{Pt}$ devices, where very similar dendrite structures can be observed, as shown in Figs. 6c and 6d [25].
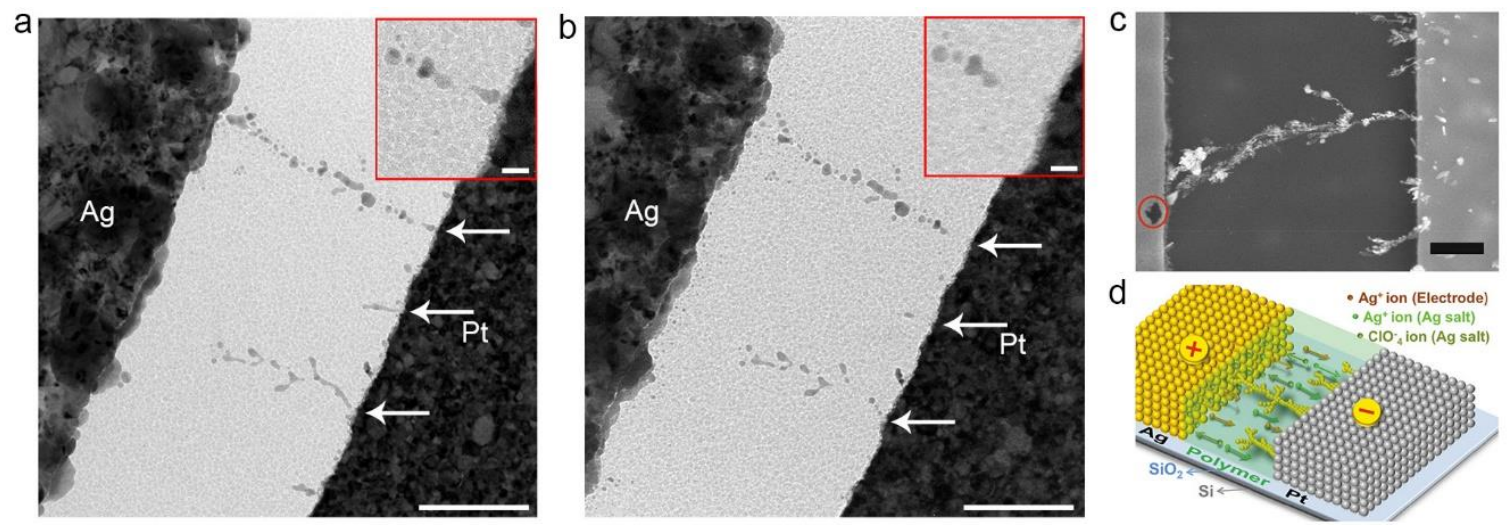

Fig. 6 Observation of conducting filament growth (a) and dissolution (b) dynamics in sputtered-SiO $\mathrm{S}_{2}$ based resistive memories. Scale bar, $200 \mathrm{~nm}$. The insets show enlarged images for the inert electrode interface. Scale bar, $20 \mathrm{~nm}$. Reprinted from [9] with permission. (c) Post-switching SEM image of an Ag/Ag-PEO/Pt device. Scale bar, $1 \mu$ m. (d) Schematic of the filament growth dynamics in an Ag/Ag-PEO/Pt device. Reprinted from [25] with permission, Copyright Wiley-VCH Verlag GmbH \& Co. KGaA

As we can see, metal filament growth in different CBRAM devices can display significantly different dynamic behaviors, and four different filament growth modes have been experimentally verified by in situ TEM observations. Based on the above analysis, it can be seen that the ion mobility in a given dielectric primarily affects the nucleation site of the filament, and this in turn decides the direction of the filament growth. Meanwhile, the redox rates are mainly responsible for the ion supply, which impact the morphology of the filament. Through such analysis, which is closely related to kinetic parameters, the diverse growth behaviors in different CBRAM systems can now be consistently explained in a generalized framework based on the same electrochemical processes. Such models may also be applicable to VCM devices. 


\section{In situ TEM observations of individual metal clusters}

As already shown in Figs. 4 and 6, in many CBRAM device systems the metal filaments are composed of individual metal clusters. This offers opportunities to further study the field-driven dynamics of individual metal nanoclusters, which is expected to provide more fundamental understanding of the switching dynamics. Utilizing the sample structure shown in Fig. 1f, Figs. 7a-d clearly show by in situ TEM observations the dynamic behavior of a single Ag nanocluster embedded in evaporated $\mathrm{SiO}_{2}$ upon application of electric field [10]. The original $\mathrm{Ag}$ cluster is gradually dissolved, while a new cluster is nucleated downstream. As the electrochemical reactions proceeded, the original cluster is fully dissolved, with the new cluster expanding in size, resulting in an overall movement of $\mathrm{Ag}$ clusters along the direction of the applied electric field (Figs. 7a-d). Similar observations were also obtained in Ref. [14]. This process can be well understood by treating the individual metal clusters as bipolar electrodes, with an effective cathode $(\delta-)$ and an effective anode $(\delta+)$ for each cluster, which experience electrochemical reduction and oxidation reactions, respectively, as schematically illustrated in Figs. $7 \mathrm{f}-\mathrm{h}$. The above cluster dynamics were found to be a universal behavior. Similar dynamics have also been observed for other metals, including $\mathrm{Cu}, \mathrm{Ni}$ and Pt. The difference between these materials lies in the electric field strength required to drive ion migration, as shown in Fig. 7e [10]. This explains why $\mathrm{Ag}$ and $\mathrm{Cu}$ are usually selected as the active electrode materials in CBRAM devices, while Pt, which requires a much higher field, is used as the inert electrode. This physicochemical process fundamentally enables ion migration in CBRAM devices and thus underpins all the above-mentioned filament dynamics. The different filament growth modes are simply different collective behaviors of the clusters under different circumstances which are described by the kinetic parameters. In this way, the different growth modes can be reconciled in the same theoretical framework. 
a

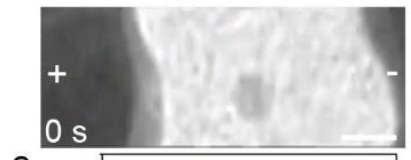

e

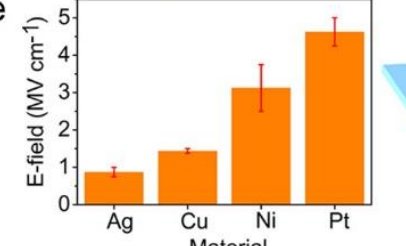

$\mathrm{b}$
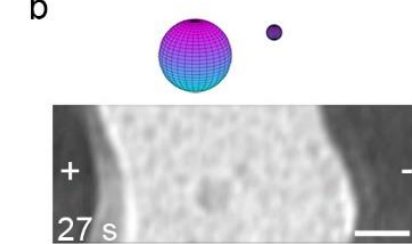

f

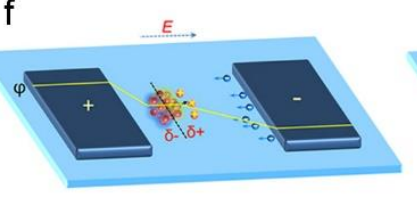

C

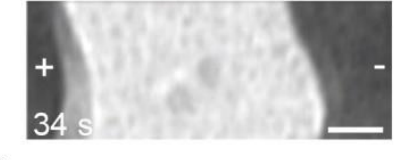

g

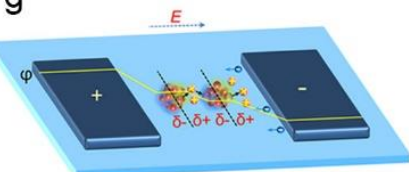

d

$10 \mathrm{~nm}$

$\mathrm{h}$

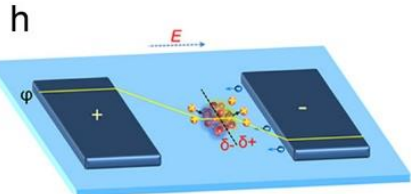

Fig. 7 (a-d) In situ TEM images along with simulation results showing dynamic behavior of a single Ag nanocluster in $\mathrm{SiO}_{2}$ under electric field. (e) Electric field required to drive the dissolution/nucleation processes for different materials. (f) Schematic of a polarized $\mathrm{Ag}$ nanocluster embedded in $\mathrm{SiO}_{2}$ under electric field, overlaid with the electric potential. (g) New cluster nucleation and formation of another bipolar electrode nearby. (h) The dissolution of the original cluster and the growth of the new cluster lead to an effective movement of a cluster along the electric field. Reprinted from [10] with permission

\section{In situ TEM observation of cyclic switching of ReRAM}

The operation mechanism of CBRAM of the formation of metallic filaments suggests the possibility of direct observation of filaments during cyclic switching in TEM. In particular, the observation of formation and rupture of the filament not only makes the mechanism of CBRAM operation clear, but also provides important information about suitable operation conditions. In addition, in order to increase the reliability of CBRAM devices, a physical model of degradation is needed, which would ensure stable operation conditions for practical applications.

\subsection{SET/RESET cycling in CBRAM}

One of the great advantages of the investigation using in situ TEM is the observation of RESET process in which all or a part of a formed filament is thought to dissipate. The principal experimental difficulty lies in distinguishing a negative result from confirmation of filament dissolution. Simultaneous observation is the only way to confirm the elimination of filaments. The direct observation of the RESET process should provide clear evidence of the mechanism of CBRAM 
switching. One of the key issues for the RESET is also the fabrication of a CBRAM cell suitable for in situ TEM.

$\mathrm{Xu}$ et al. first reported clear observation of the RESET process in the W/AgS $/ \mathrm{Ag}$ system, in which the $\mathrm{AgS}_{2}$ solid-electrolyte layer was contacted with an $\mathrm{Ag}$ nanowire [18]. The counter electrode was a movable W tip of an STM placed within the TEM. CBRAM cells fabricated with needle-type top and bottom electrodes allow easy observation of structural changes in the surface because every position of the bottom electrode surface covered by $\mathrm{AgS}_{2}$ can be used as a CBRAM cell by attaching the tungsten STM tip. In this case, reasonably clear hysteresis in current-voltage (I-V) characteristics was observed in the TEM. As shown in Fig. 8, a relatively large on/off current ratio of up to five orders of magnitude was achieved. EDS and HRTEM lattice images were employed to investigate the formation and rupture of the conductive filament by clarifying the lattice structures and material composition. It was confirmed that a part of the initial high resistance argentite phase $\mathrm{AgS}_{2}$ was changed to a mixture of low-resistance acanthite-phase $\mathrm{AgS}_{2}$ and metallic Ag nanoparticles during SET. Part of the low resistance Ag-rich area was detected to change to the argentite phase during RESET.

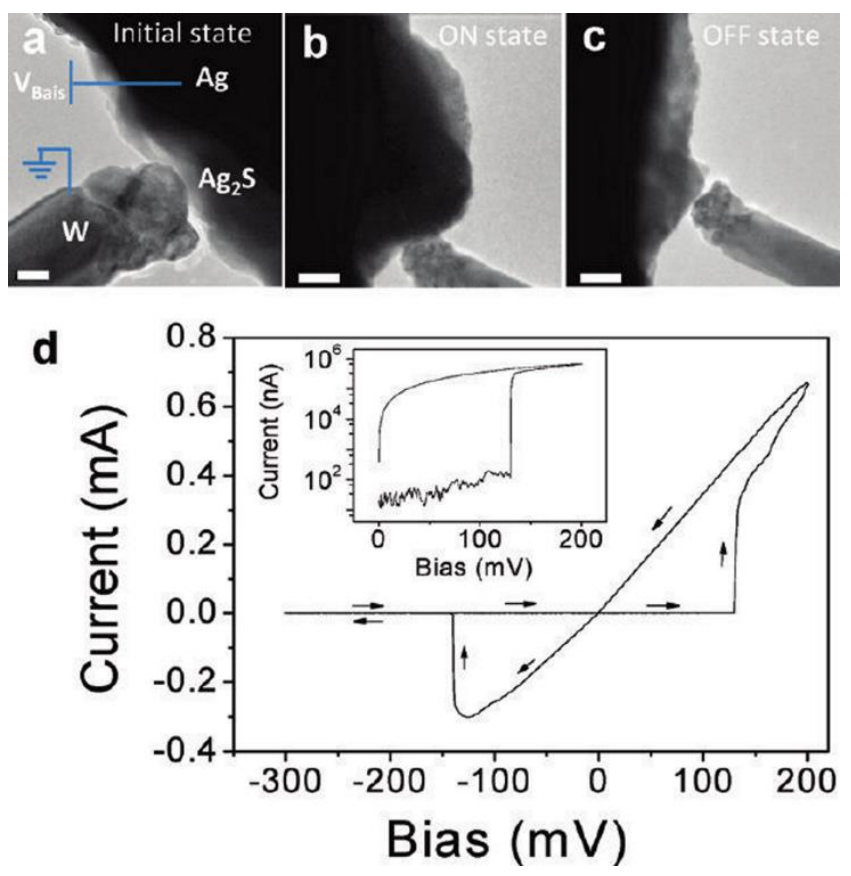

Fig. 8 TEM images of the initial off-state (a), the on-state (b), and after returning to the off-state (c). Scale bars are $20 \mathrm{~nm}$ (a), and $50 \mathrm{~nm}$ (b) and (c). (d) I-V hysteresis curve. Inset: I-V curve (positive range) on a semilog plot. Reprinted from [18] with permission 
A similar analysis was reported by Fujii et al. for the PtIr/Cu-GeS/PtIr system in which both of the top and bottom electrodes were also needles [17, 30]. The bottom electrode was a PtIr needle covered by a thin $\mathrm{Cu}-\mathrm{GeS}$ solid electrolyte formed by magnetron sputtering. The top electrode was a movable PtIr STM tip. Clear TEM video images in which $\mathrm{Cu}$-like filaments grew from the bottom electrode during the SET process and disappeared toward the electrode during the RESET process were observed, together with $I-V$ hysteresis. The timings of the growth and elimination of a filament corresponded to the resistance changes, which are clear evidence of the SET and RESET operations of CBRAM. EDS and selective area diffractometry (SAD) revealed that the filament contains a large amount of metallic $\mathrm{Cu}$.

More sophisticated CBRAM samples applicable to in situ TEM were fabricated by Liu et al. by employing skillful FIB fabrication, as shown in Fig. 9 [13]. A sample stage was made at the top of a tungsten needle cut by FIB (Fig. 9a). 100-nm-scale stacked $\mathrm{Ag}$ (or $\mathrm{Cu}$ )/ $\mathrm{ZrO} 2 / \mathrm{Pt} / \mathrm{Ti} \mathrm{CBRAM}$ cells were also formed using FIB cutting and milling. SET and RESET I- $V$ characteristics were measured in the TEM, as shown in Fig. 9c and 9d. Detailed observations, together with the EDS analysis, confirmed the formation of $\mathrm{Ag}$ or $\mathrm{Cu}$ filaments in the $\mathrm{ZrO}_{2}$ electrolyte during the SET process. The dissolution of the filament toward the cathode was observed during RESET.

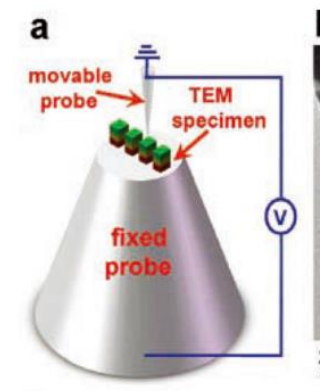

C

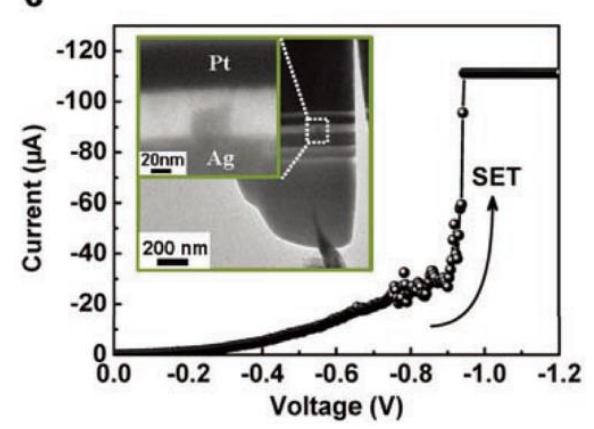

b
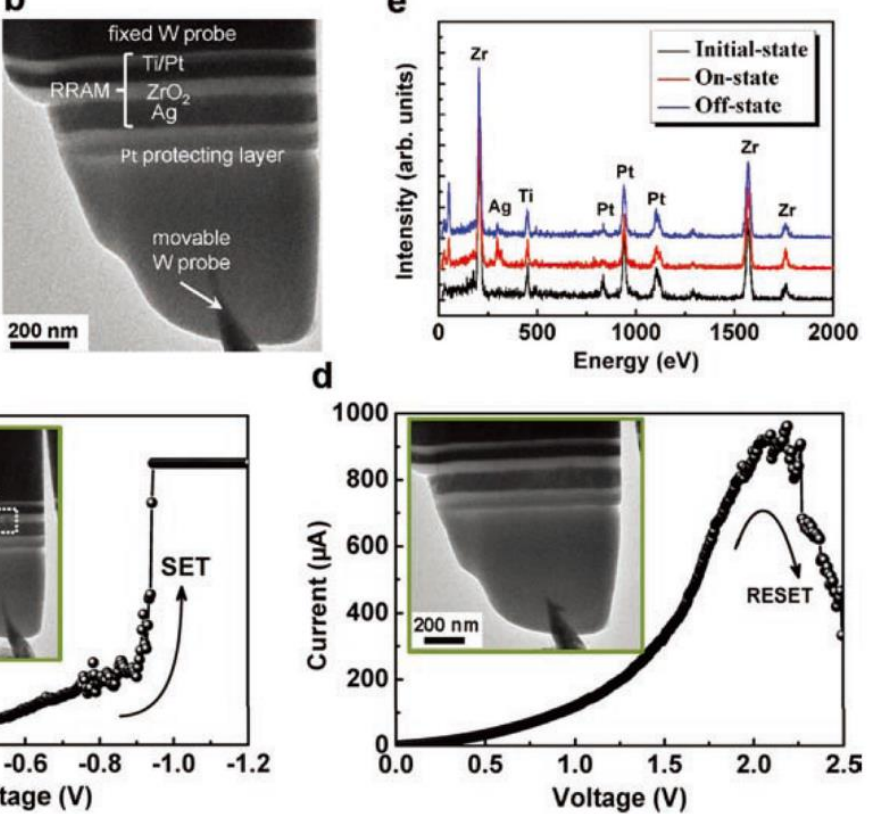

Fig. 9 (a) Schematic of an in situ TEM experimental setup. (b) Cross-sectional TEM image of a fresh $\mathrm{Ag} / \mathrm{ZrO} 2 / \mathrm{Pt}$ memory device. (c) I-V curve during SET operation with image after SET and during RESET operation (d) with image 
after RESET. (e) EDS spectra at the initial-, ON- and OFF-states. Reprinted from [13] with permission, Copyright Wiley-VCH Verlag GmbH \& Co. KGaA

Direct observation of a pair of SET/RESET operations enabled observation of the formation and rupture of the filament. It should be noted that almost all reports of in situ TEM analysis confirm a negligible electron-beam radiation effect on the SET/RESET switching characteristics.

\subsection{Multiple switching cycles}

The observation of multiple resistance switching cycles is necessary because repeatable write and erase cycles are essential for RAM. A crucial point for the measurement is to ensure stable positioning of the STM probing system working in TEM, which can be achieved only by continuous and steady efforts towards improvement. The observation of the cyclic filament formation and rupturing makes allows precise relations between the operation condition and the filament formation to be revealed, enabling further understanding of the switching mechanism.

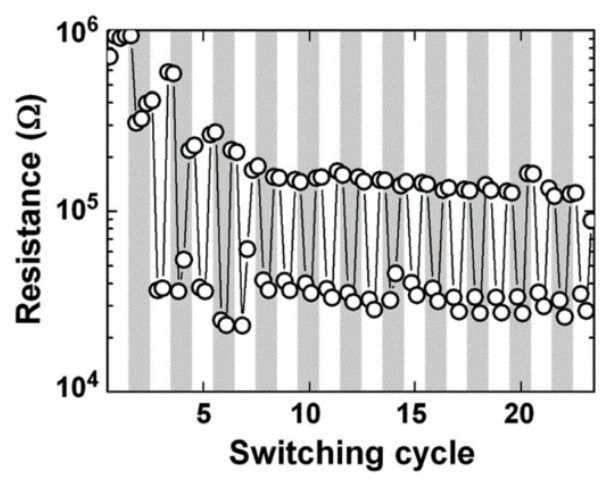

Fig. 10 Cyclic endurance properties of the Pt/MoOx/TiN CBRAM system. Reprinted from [31] with permission

The first CBRAM cyclic switching characteristics were observed by through the use of the $\mathrm{Pt} / \mathrm{MoO}_{\mathrm{x}} / \mathrm{TiN}$ CBRAM system in which the $\mathrm{MoO}_{\mathrm{x}}$ layer was used as a solid electrolyte [31-34]. Needle-like stacked CBRAM samples were formed on a Si wafer using an ion-shadow process [35] and measured in a TEM using a movable STM PtIr counter electrode. More than 20 cycles of SET/RESET operations were successfully achieved, as shown in Fig. 10 [31]. TEM time-resolved images in which $\mathrm{Cu}$-like filaments were cyclically formed and dissolved between the top and bottom electrodes were reported. The formation of $\mathrm{Cu}$ filaments occurred from the cathode (TiN) toward the 
anode $(\mathrm{Cu})$, fitting well with a model in which positive $\mathrm{Cu}$ ions move from anode to cathode and deposit as a filament [33]. In the RESET process, however, $\mathrm{Cu}$ filaments shrank towards the $\mathrm{Cu}$ electrode, as shown in Fig. 11, which is not consistent with the Cu re-ionizing model [36, 37].

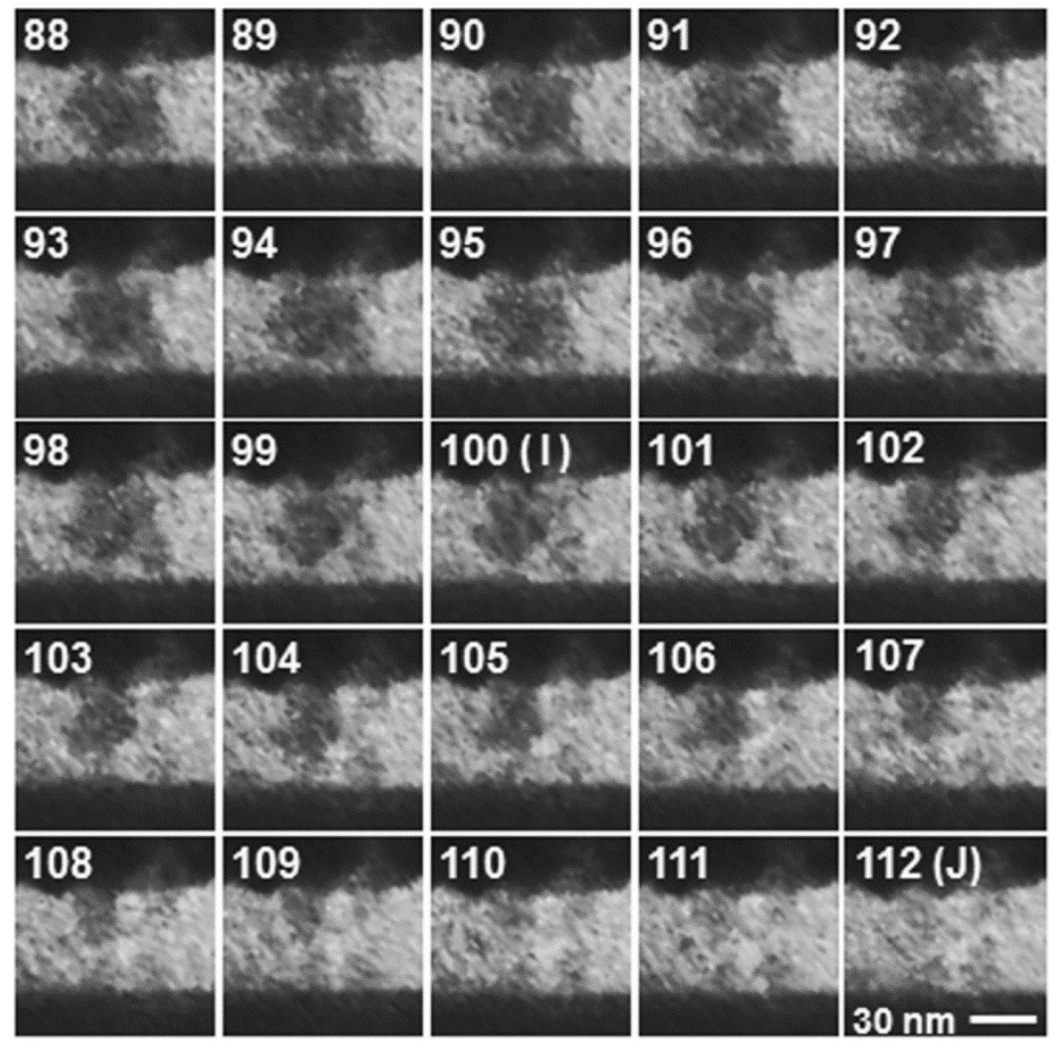

Fig. 11 TEM images of filaments in the $\mathrm{Pt} / \mathrm{MoO}_{\mathrm{x}} / \mathrm{TiN}$ CBRAM system during the RESET process, extracted from a video. Numbers indicate the order of video frames. The interval between video frames was 30ms. The top dark, middle bright, and the bottom dark areas correspond to $\mathrm{Cu}$, MoOx, and TiN layers, respectively. Reprinted from [33] with permission

An important finding in cyclic operation is the relation between the compliance current and the size of the conductive filament. As predicted in previous work which shows a clear relation between the compliance current and resistance, an increase in filament diameter with increasing compliance current was confirmed. Although an unvarying filament position may naively be expected during cyclic operation, filaments were sometimes formed at different positions [33]. To clarify this phenomenon, further investigation of the time dependent SET/RESET operation, including over/under SET and/or RESET [31] is necessary.

Similar results were achieved by Hubbard et al. for $\mathrm{Pt} / \mathrm{Al}_{2} \mathrm{O}_{3} / \mathrm{Cu}$ CBRAM through the use of 
specially designed lateral cells in which $\mathrm{Pt}$ and $\mathrm{Cu}$ electrodes were formed opposite each other across a thin $\mathrm{Al}_{2} \mathrm{O}_{3}$ layer on a SiN membrane [15]. More than ten cycles of SET/RESET switching were performed in TEM. In the SET operation, a $\mathrm{Cu}$ filament growth from the Pt electrode to the $\mathrm{Cu}$ one was observed, time-resolved TEM images in Fig. 12 show. A partially cut $\mathrm{Cu}$ filament at the $\mathrm{Cu}$ electrode side was observed after RESET. These results are consistent with the standard model of CBRAM operation [36, 37].

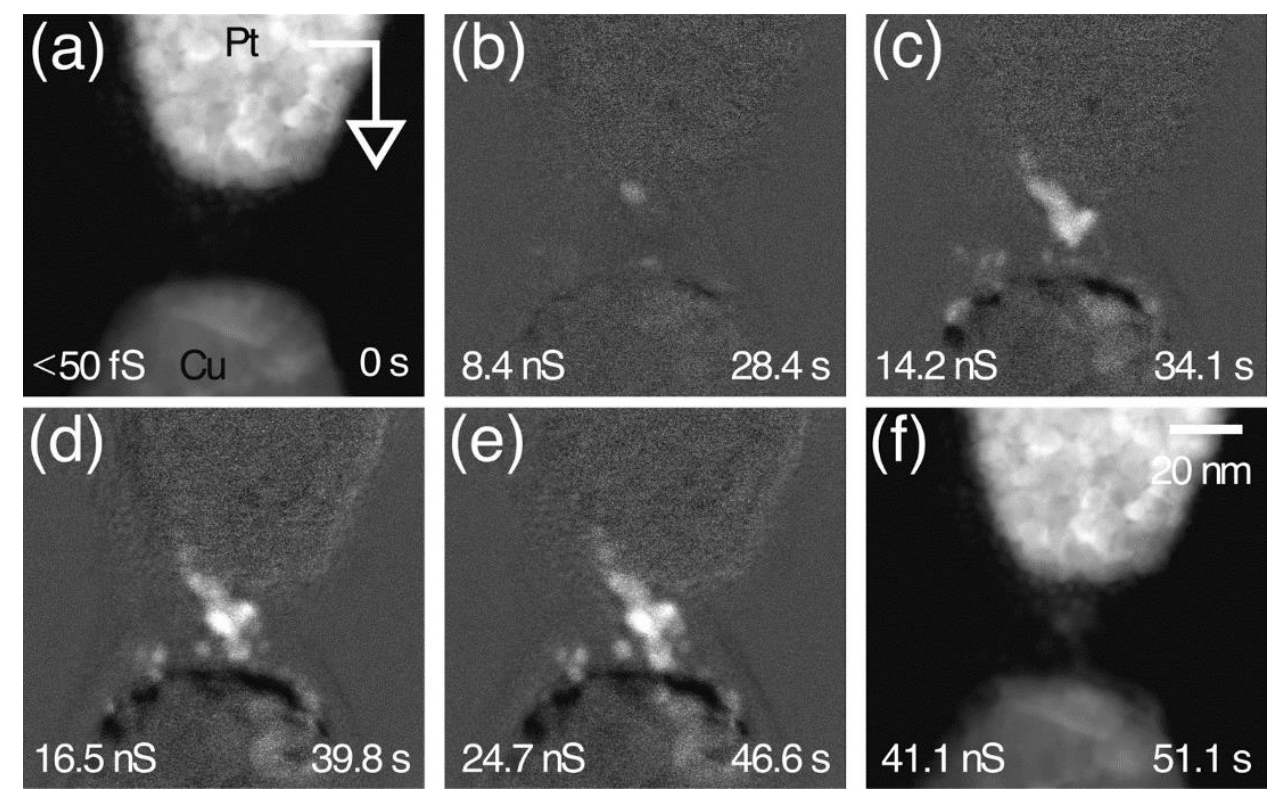

Fig. 12 Time-resolved images of filament growth in a $\mathrm{Pt} / \mathrm{Al}_{2} \mathrm{O}_{3} / \mathrm{Cu}$ CBRAM before (a) and after (f) filament formation. The filament spanning the gap is clearly visible in part f. Reprinted from [15] with permission

\subsection{Device characterization \& reliability issues analyzable by in situ TEM}

It is well known that one of the key uses of TEM in semiconductor industries is in failure analysis of devices and circuits. If we can operate ReRAM devices in a TEM, failure processes will be observed, which may enable us to determine their origin. For the purpose, many cyclic operations should be run in the TEM.

Arita et al. investigated a type of endurance failure for a $\mathrm{Cu} / \mathrm{WO}_{\mathrm{x}} / \mathrm{TiN} \mathrm{CBRAM}$ [12]. Ten cycles of resistance switching were tested by in situ TEM by increasing the compliance current from 20 to $300 \mu \mathrm{A}$ step by step, which resulted in degradation (reduction of resistance) of the high resistance states (HRS). This test corresponds to so-called accelerated current (voltage) stress tests [38]. The 
TEM images at HRS after the RESET operation (Figs. 13a-h) showed that the $\mathrm{WO}_{\mathrm{x}}$ layer (bright contrast region) seemed to be thinner. These results clearly showed that the origin of HRS degradation was $\mathrm{Cu}$ deposition at the top and bottom electrodes.

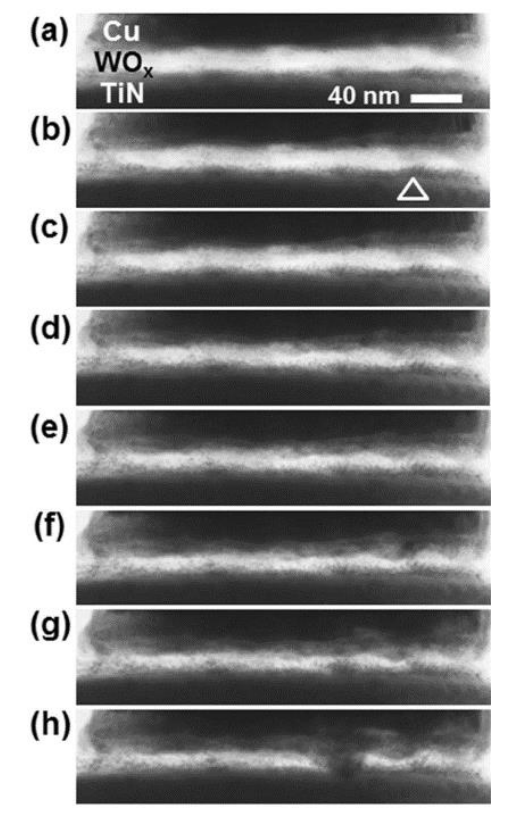

Fig. 13 TEM images of a $\mathrm{Cu} / \mathrm{WO}_{\mathrm{x}} / \mathrm{TiN}$ CBRAM in the initial state (a), and images after continuous RESET with a SET compliance current of $20 \mu \mathrm{A}$ (b), $50 \mu \mathrm{A}$ (c), $100 \mu \mathrm{A}$ (d), $150 \mu \mathrm{A}$ (e), $200 \mu \mathrm{A}$ (f), $250 \mu \mathrm{A}$ (g), and $300 \mu \mathrm{A}(\mathrm{h})$. Reprinted from [12] with permission

An extreme example of stable cyclic operation was achieved using a CuTe-based CBRAM similar to that developed as a 16 Mbit memory by Sony and Micron [40]. I-V hysteresis loops of more than 60 cycles, and pulse switching operation of 100000 cycles were achieved by Kudo et al. together with the TEM images of filaments as shown in Figs. 14a and b, respectively [39, 41]. The samples used for in situ TEM were fabricated using FIB; an example is shown in the inset of Fig. 14a. In addition, retention of the memory state for over three months was obtained. These results suggest that every electrical measurement that is usually carried out using a probe station can be performed in the TEM. 

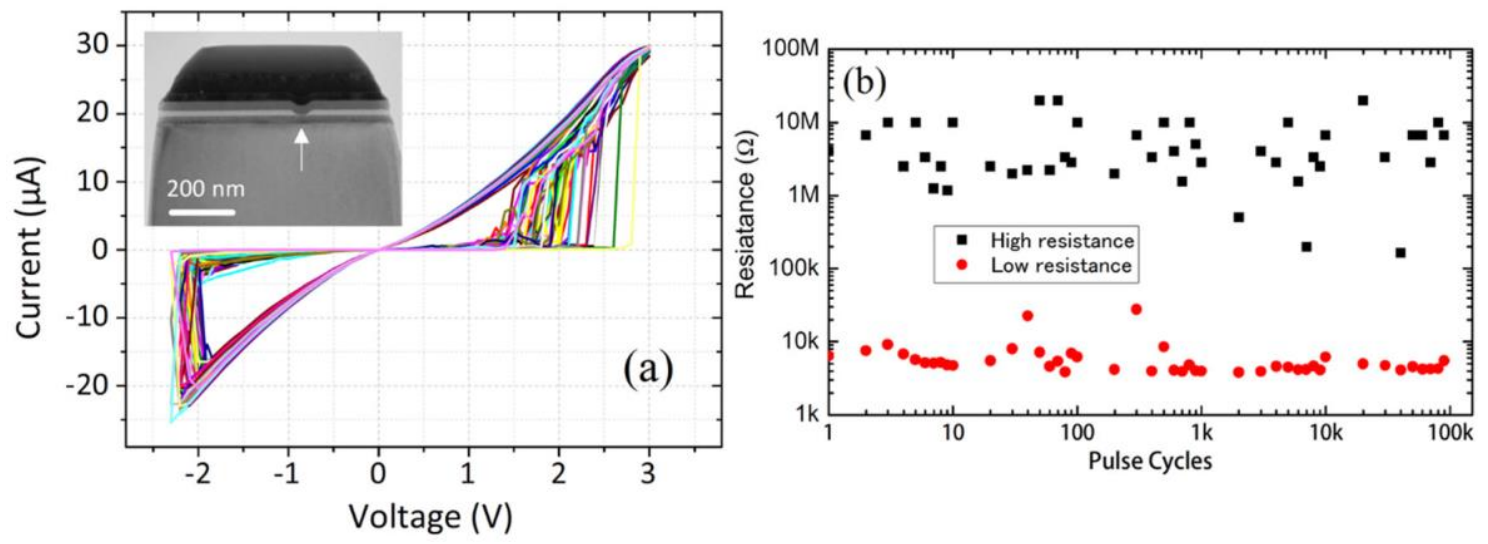

Fig. 14 (a) I-V hysteresis characteristics of more than 60 SET/RESET cycles in a CuTe-based CBRAM, with a TEM image of the measured sample, and (b) SET/RESET pulse cycles on the 30-nm cell measured in a TEM. Reprinted from [39] with permission

The results described above are clear evidence that in situ TEM is a powerful tool to analyze the reliability of CBRAM, because the images before and after changes or degradation can be observed directly. However, one of the difficulties in using in situ TEM is that the set-up of the system is complicated to use, and sample preparation is challenging, which makes the turn around time of evaluation long. If we can intentionally introduce defects or impurities suggesting degradation in the TEM sample, cause and effect relationships will be more easily clarified.

\section{In-situ TEM of filamentary oxide ReRAM}

The ReRAM operations of oxides may be classified into two categories: filamentary and interface switching, while bulk effects like Mott transition are also discussed. Conductive filaments $(\mathrm{CFs})$ of oxygen vacancies formed in the insulating oxide layer play an important role in the filamentary ReRAM, while electronic states near the electrode-oxide interface contribute to the operation in the interface switching $[2,42,43]$. In this section, in situ TEM of filamentary oxide ReRAMs is surveyed. In this case, a soft-breakdown process called electroforming is required prior to the SET/RESET operation. In the usual case, the CF template is formed in the initially highly resistive switching layer, giving the initial LRS. Resistance switching is understood to occur by rupture (RESET) and growth (SET) of the CF through thermochemical or electrochemical change of the oxide valence state (TCM or VCM). 
Electroforming can generate significant stresses, which can result in large-scale movement of oxygen within samples [44], and the ReRAMs can occasionally fail even if instrumental current compliance is set [45]. This is fatal for tiny in-situ TEM samples exposed to the vacuum. Fujii et al. investigated the electroforming process of $\mathrm{NiO}$ with a series resistor of $1 \mathrm{k} \Omega$ [46] to avoid this issue. As shown in Figs. 15a-b, they demonstrated sudden formation of the CF. The CF contained less oxygen than the bulk of the oxide (Fig. 15c-d) [47], and its diameter decreased by reducing the current. However, the subsequent RESET was not achieved because the oxygen liberated during electroforming escaped into the vacuum. Reports of in-situ electroforming are rare. This is because control of power dissipation during electroforming is difficult, and current overshoot is hard to prevent. There was a report to realize gradual electroforming, in which oxygen vacancies were introduced by the TEM electron beam [48]. Another way is the detection of the switching region after electroforming, using an FIB. Though it is time consuming to find the CF region, it can be a way to investigate the SET/RESET operations excluding the experimental difficulties.
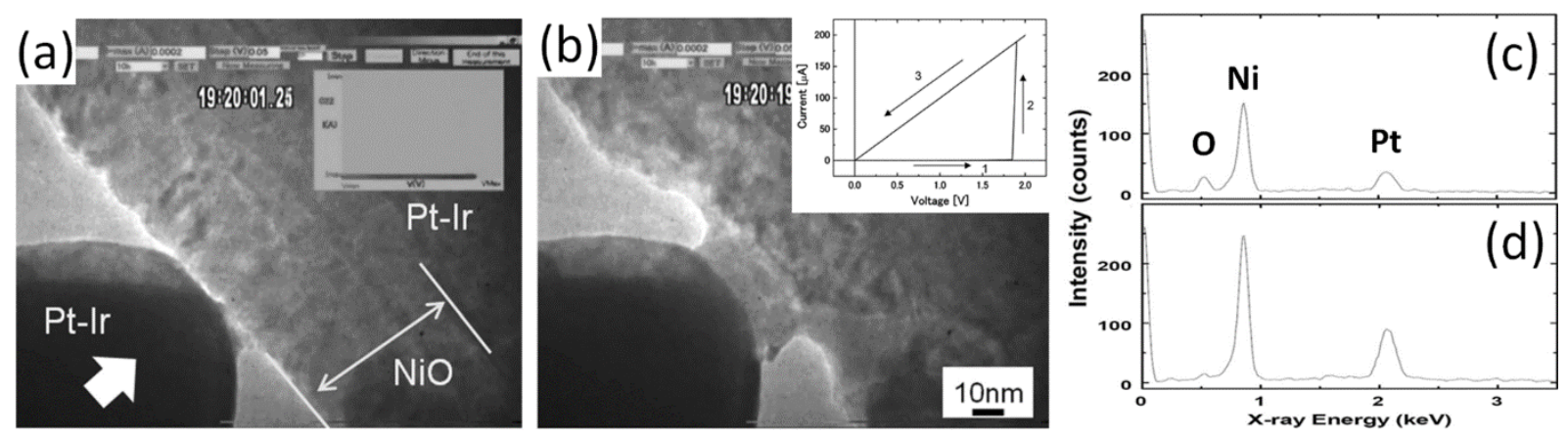

Fig. 15 (a-b) In-situ TEM video images before and after electroforming of PtIr/NiO/PtIr. Inset in (b) is the $I$ - $V$ graph of electroforming. (c-d) EDS spectra from the CF and the neighboring region, respectively. Reprinted from [46, 47] with permission

In the following section, successful examples of in situ observations are listed. After ex-situ and in situ TEM of $\mathrm{Pt} / \mathrm{TiO}_{2} / \mathrm{Pt}$, it was confirmed that a $\mathrm{CF}$ of the conductive $\mathrm{Ti}_{4} \mathrm{O}_{7}$ Magnéli phase contributes to the operation [11, 49]. Kwon et al. performed in situ RESETs using a SET sample containing the Magnéli CF. The resistance increased (Fig. 16a), and the superlattice reflections of the Magnéli phase correspondingly disappeared (Figs. 16b-c) [11]. In a $\mathrm{Pt} / \mathrm{NiO} / \mathrm{TiO}_{2} / \mathrm{Pt}$ stack ReRAM, it was reported that $\mathrm{CF}$ rupture at the interface between two oxides, and the method to localize the RESET position and to achieve quick RESET was discussed [50]. Serial in situ TEM is expected to 
shed light on the dynamics of this process.
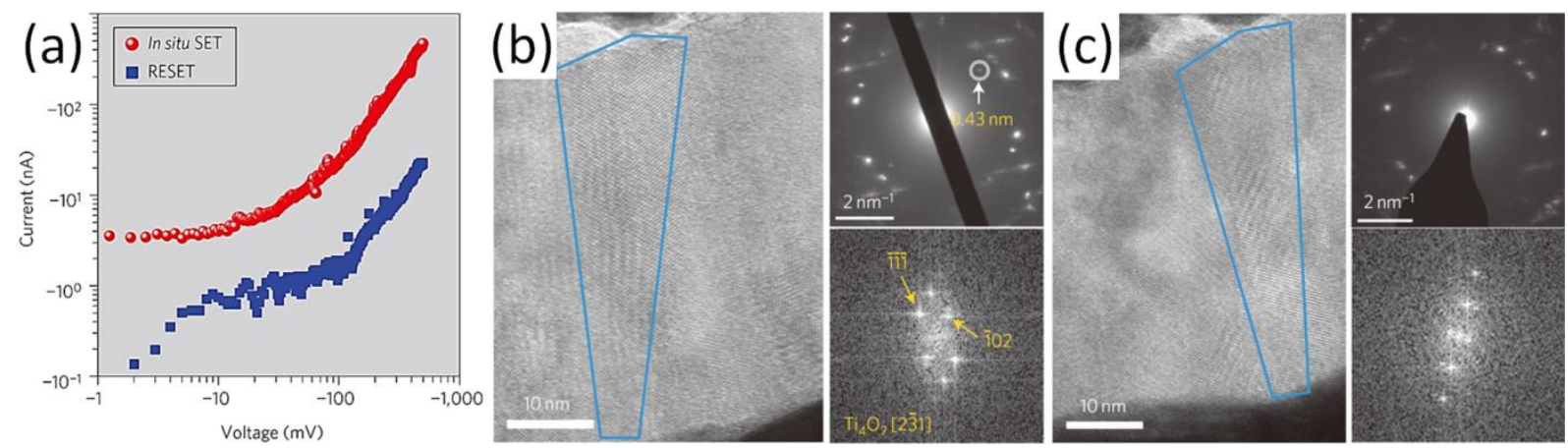

Fig. 16 In situ RESET of Pt/TiO $/$ /Pt. (a) I-V graph. (b) HRTEM image, electron diffraction pattern and FFT pattern of the image before the RESET operation, and (c) those after RESET. Reprinted from [11] with permission

In situ TEM of the unipolar $\mathrm{Pt} / \mathrm{ZnO} / \mathrm{Pt}$ by $\mathrm{Chen}$ et al. was a rare example where the electroforming was achieved with subsequent in situ switching operations (Fig. 17a) [51]. By applying a negative voltage to the top electrode (TE) of the initial state (Fig. 17b), dark contrast appeared in the $\mathrm{ZnO}$ layer near the electrode. Afterwards, a filament like contrast grew from the TE (Fig. 17c) and the LRS was achieved when it reached the bottom electrode (BE) (Fig. 17c). The researchers performed EELS measurements and concluded that the $\mathrm{CF}$ was $\mathrm{Zn}$-rich $\mathrm{ZnO}_{1-\mathrm{x}}$ (or $\mathrm{Zn}$ ). This CF growth can be understood to be caused by migration of oxygen vacancies along the electric field and their accumulation. EDS data indicated lower oxygen content near the cathode (TE). In the RESET process, the CF ruptured at the narrowing position where Joule heating is significant (Fig. 17e). The remaining section of the $\mathrm{CF}$ at the BE side shrank toward the BE (arrow in Fig. 17f). Thermochemical reactions accompanied by oxygen migration is thought to contribute this RESET operation, as is widely expected for unipolar ReRAM. Actually, the HRTEM image from the ruptured position showed $\mathrm{ZnO}$ fringes (Fig. 17g) while the image from the $\mathrm{CF}$ was of $\mathrm{Zn}$ (Fig. 17h). Unfortunately, the resistance became stuck in the LRS without further switching after several switching operations. This may be caused by loss of oxygen into the TEM vacuum. 

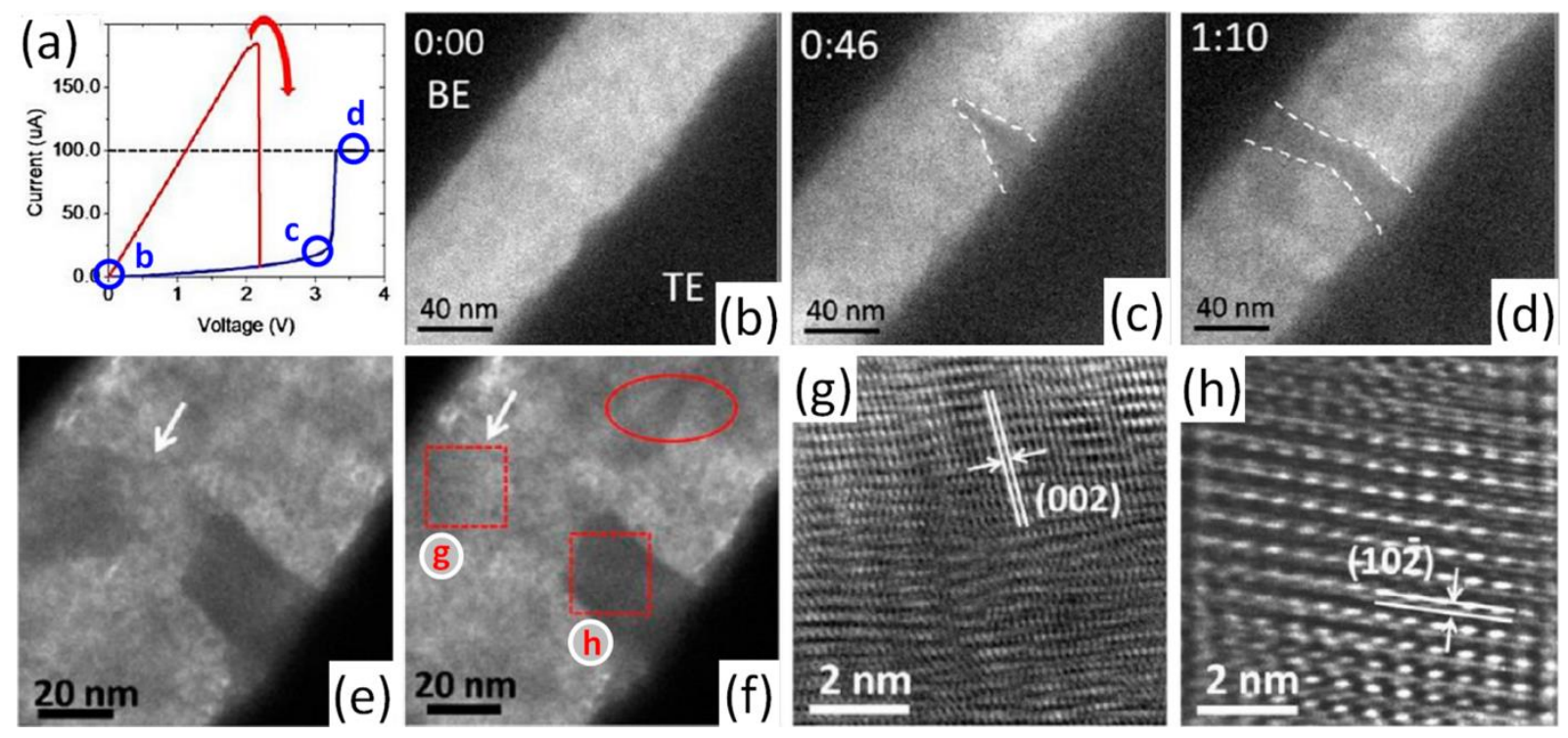

Fig. 17 In situ electroforming and RESET of Pt/ZnO/Pt where the TE was negatively biased while the BE was grounded.

(a) $I-V$ graph. Corresponding (b-d) electroforming and (e-f) RESET images extracted from a video. (g-h) HRTEM images from square region in (f). The red ellipsoid is relevant only in the original reference. Reprinted from [51] with permission

Tantalum oxides are widely investigated for CMOS-compatible ReRAM devices. Park et al. reported in-situ switching of the $\mathrm{Pt} / \mathrm{SiO}_{2} / \mathrm{a}-\mathrm{Ta}_{2} \mathrm{O}_{5-\mathrm{x}} / \mathrm{a}-\mathrm{TaO}_{2-\mathrm{x}} / \mathrm{Pt}$ structure (Fig. 18a) where the $\mathrm{SiO}_{2}$ layer was included to reduce the switching current [52]. They achieved bipolar operation, and an on/off resistance ratio larger than 100 was realized (Fig. 18b). Nano-scale CFs introduced during electroforming were partly annihilated and re-formed in the RESET and SET processes, respectively (Fig. 18c). Based on the EELS-STEM results (Fig. 18d), the CF was concluded to be metallic TaO in the LRS and an insulating $\mathrm{Ta}_{2} \mathrm{O}_{5}-\mathrm{TaO}_{2}$ mixed valence compound in the HRS. After careful analysis of HAADF-STEM data, they claimed that switching occurred within a section of the CF between the uppermost Pt and a-TaO ${ }_{2-x}$. Using HAADF-STEM and ex situ Auger measurements, they discussed the migration of oxygen vacancies driven by electric stress. Realization of multiple in situ switching cycles is one of the important results of this work, which is useful for degradation tests of ReRAM devices. 

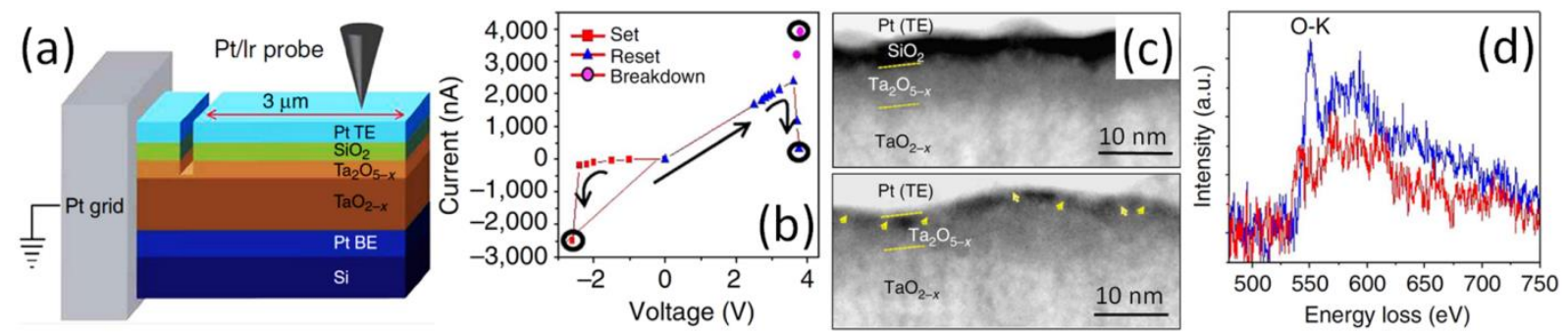

Fig. 18 (a) Schematic drawing of an in situ $\mathrm{Pt} / \mathrm{SiO}_{2} / \mathrm{a}-\mathrm{Ta}_{2} \mathrm{O}_{5-\mathrm{x}} / \mathrm{a}-\mathrm{TaO}_{2-\mathrm{x}} / \mathrm{Pt}$ TEM sample. (b) In situ SET/RESET I-V graph. (c) HAADF-STEM images of HRS and LRS. (d) STEM-EELS spectra from the CF of HRS and LRS. Reprinted from [52] with permission

There have been reports of complex VCM/CBRAM behavior in systems involving metallic CFs other than the more conventional $\mathrm{Cu}$ and $\mathrm{Ag}$ used for CBRAMs. Wu et al. investigated the unipolar $\mathrm{Ni} / \mathrm{HfO}_{2} / \mathrm{SiO}_{2} / \mathrm{Si}$ system in which $\mathrm{Ni}$ was positively biased [53]. On electroforming with a compliance current of $10 \mu \mathrm{A}$, no change was seen in the switching region. In subsequent operation at $100 \mu \mathrm{A}$, the resistance decreased further (SET, Fig. 19a), and Ni penetrated the $\mathrm{HfO}_{2}$ and $\mathrm{SiO}_{2}$ layers and reached the $\mathrm{Si}$, forming a silicide (Fig. 19b). It was hypothesised that the $\mathrm{CF}$ of oxygen vacancies was made during electroforming, and a metallic Ni CF punched through the oxide layers during the SET process. Migration of Ni was clearly opposite to electromigration. During the RESET process as the resistance increased (Fig. 19c), the Ni CF vanished from the oxide layer (Fig. 19d). The Ni CF thus contributed to the switching but was not the whole story. As is widely accepted, an increase of the switching current enhances the retention of the LRS [54]. However, this may increase the probability of CFs getting material from the electrode. Careful investigation of these processes will be useful for understanding device failure.
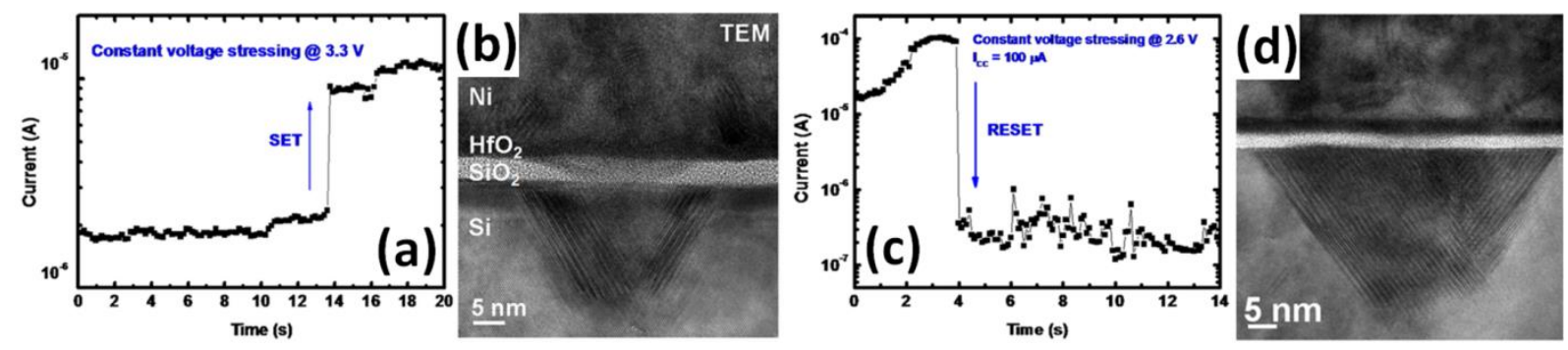

Fig. $19 \mathrm{SET}$ and RESET operation of unipolar $\mathrm{Ni} / \mathrm{HfO}_{2} / \mathrm{SiO}_{2} / \mathrm{Si}$. (a-b) Time dependence of current and HRTEM image during SET, and (c-d) those during RESET. Reprinted from [53] with permission

In the examples listed in this section, the CFs formed of oxygen vacancies play key roles in 
resistance switching. Therefore, distributions of the oxygen vacancies and accompanying changes of the crystal structure should be investigated carefully by in situ TEM. Consequently, various experiments like TEM, HRTEM, HAADF-STEM, EDS, EELS and SAD have been performed [see, for example, 44]. These investigations are valid also for research studying electronic devices other than ReRAMs. For example, Li et al. performed in-situ EELS-STEM and investigated the distribution of oxygen vacancies during electrical stressing to understand the properties of Flash memories [55]. Their state is essentially the same as that of ReRAMs just before electroforming. Searching of in-situ TEM reports in other related research fields will therefore give interesting information to researchers developing ReRAM devices.

At the end of this section, other examples are briefly described. The Perovskite-type complex oxides are widely known ReRAM materials. In contrast to above studies of filamentary switching, no clear TEM image change was seen in the in situ switching of polycrystalline $\operatorname{Pr}_{0.7} \mathrm{Ca}_{0.3} \mathrm{MnO}_{3}$ (PCMO) [56]. To understand details of the switching layer, epitaxial films have been investigated for in situ TEM. For example, in situ TEM/EELS of a Pt/La $0.85 \mathrm{Sr}_{0.15} \mathrm{TiO}_{3}(\mathrm{LSTO}) / \mathrm{SrTiO}_{3}: \mathrm{Nb}$ epitaxial film was performed [57]. By comparing with reference films, it was reported that the oxygen content at the Pt/LSTO interface decreased after electrofroming and SET, and increased at RESET. On the other hand, in the $\mathrm{Au} / \mathrm{PCMO} / \mathrm{SrTi}_{0.99} \mathrm{Nb}_{0.01} \mathrm{O}_{3}$ (STNO), only a faint change of oxygen content at the PCMO/STNO interface after SET was detected [58]. Nevertheless, in situ electron diffraction patterns from PCMO indicated detwinning of the pesudo-cubic PCMO as well as structural transition to a long-range ordered phase after RESET. This change in the bulk PCMO film was claimed to contribute the resistive switching besides the interface dominated switching seen in PCMO with non-noble metal electrodes. The appearance and/or disappearance of the ordered phase was reported in other references for PCMO, and oxygen vacancy ordering and the electronic band bending at the electrode/PCMO interface were discussed in relation to switching [59, 60]. The rare-earth oxide $\mathrm{CeO}_{2} / \mathrm{STNO}$ epitaxial film investigated by in situ HRTEM, SAD as well as EELS is another example [61]. In this case, a superstructure $\mathrm{Ce}_{2} \mathrm{O}_{3}$ caused by oxygen vacancies appeared at SET and disappeared at RESET. This may achieve the filamentary switching as in the $\mathrm{TiO}_{2}$-base $\mathrm{ReRAM}$. An interesting result was reported for a $\mathrm{TiO}_{2}$ (rutile) single crystal sandwiched between tungsten and TiN electrodes [62]. At electroforming, the Magnéli phase developed at the $\mathrm{W} / \mathrm{TiO}_{2}$ interface and 
extended towards the $\mathrm{TiO}_{2} / \mathrm{TiN}$ interface but with a $50 \mathrm{~nm}$ thick Magnéli phase-free zone near TiN. At SET, the Wadsley defects passed through this free zone, which are planar defects of oxygen vacancies. The defects retracted at RESET. This defect may behave as the CF, though further investigation is required.

While various oxides have been reported to show ReRAM switching and several switching mechanisms have been proposed, the number of in situ TEM reports are still not so much. Nevertheless it is hard to detect the migration and accumulation of oxygen vacancies and related crystallographic change in local areas, further recording of in situ TEM results are expected for deep understanding of this type of ReRAMs.

\section{Scanning tunneling microscopy for the investigation of resistance switching phenomena}

Conventionally, resistance switching (RS) devices use thin dielectric films, sandwiched between conductive electrodes, as active layers whose conductivity can be electrically tailored [2]. The physical structure of these materials is fundamental to their electrical properties and significant correlation has been demonstrated between structural reconfiguration and changes in device resistance $[29,44]$.

As the field of resistance switching grows, a diversity of characterisation methods are needed in order to study the relationship between nanoscale structures and electrical behaviour. Highly confined nanoscale features, such as conductive filaments and localised phase changes, can be responsible for variations in the conductive state of the active layer [9, 63]. Scanning probe microscopy (SPM) enables the probing of features on the nanoscale, along with the facility for spectroscopic measurements. However, the generally insulating nature of the pristine oxide materials used in resistance switching presents particular challenges for scanning probe microscopies that measure variations in conductivity.

Generally, the top electrode of a resistance switching device is fixed and used as a good contact for electrical operation. However, this complicates the systematic study of the active layer as it is then inaccessible beneath the electrode, which must be removed in order to probe the layer below. 
An advantage of SPM as applied to RS devices is that the behaviour of the active layer can be studied without the presence of a top electrode [64-69], allowing researchers to further their understanding of switching materials - both the pristine states and those formed after electrical bias. The standard technique for such studies is conductive atomic force microscopy (CAFM), used for the first time by Murrell et al. to investigate local dielectric properties of $\mathrm{SiO}_{2}$ thin films [64] and soon extended to a wide range of material systems [65]. Here, the conductive tip of the CAFM cantilever acts as a microelectrode with which both read and write processes can be performed.

CAFM is readily applicable to many materials and aside from classical conductance and switching measurements, more advanced experiments have been performed. An interesting example is conductance tomography, in which electrical conductivity mapping is combined with ablation of surface layers in order to realise three dimensional maps of conductive filaments [63].

However, CAFM has several disadvantages. Due to the nature of contact mode measurements, mechanical deformation of the surface morphology is possible, particularly on soft materials or with high loading forces [67]. The switching properties may also be strongly dependent on the loading force, as e.g. Singh et al. demonstrated for $\mathrm{Cu}_{2} \mathrm{O}$ [68]; depending on the used loading force either unipolar or bipolar switching was observed. Furthermore, most CAFM cantilevers use standard silicon tips, coated with a thin metal or doped diamond layer. This increases the diameter of the tip apex (typically $>10 \mathrm{~nm}$ ), limiting the lateral imaging resolution and therefore the minimum switching area. Local heating effects at higher currents may also damage or remove the coating, affecting the conductivity and resolution of the tip.

STM is a powerful alternative for CAFM and addresses most of these problems. STM is a valuable tool in probing nanoscale features and electrical behaviour simultaneously, as the probe can act as both an electrode and a map.

STM uses an atomically sharp metallic needle to scan a conductive sample surface, while a voltage is applied between the needle and sample. The field between the tip and the sample allows the local redox and ion transport processes at the heart of RS to be initiated and observed [69]. Unlike in contact-mode CAFM, there is no mechanical contact between the tip and the surface, thus mechanical deformation of the sample is excluded. By holding either the needle height or tip-sample 
current constant and measuring the other variable, it is possible to map both the topography of the sample and its conductivity.

Electrons tunnel through the vacuum gap between the tip and the sample surface. By using quantum mechanical perturbation theory under the assumption of a spherical tip apex with the radius $r$ the tunnelling current $I_{T}$ can be approximated for low bias voltages $U_{B}$ by the following equation:

$$
I_{T} \sim U_{B} \cdot \rho\left(E_{F}\right) \cdot e^{-2 k \cdot(r+z)}
$$

The exponential dependence of the measured tunnelling current on the tip-sample distance $z$ results in a nearly exclusive contribution of the outermost atom of the STM tip to the tunnelling process. Thus, STM offers a greatly increased spatial resolution compared to CAFM. Furthermore, the dependence of tunnelling current on the local electron density $\rho\left(E_{F}\right)$ at the Fermi level provides information on the electronic properties of the sample, which can be studied by scanning tunnelling spectroscopy (STS). However, the additional dependence on the electronic states of both the sample and the tip can complicate the interpretation of STM images due to the mixture of electronic and topographic information.

Although not the focus of this review, it is of note that STM measurements in the extended field of resistance switching led to the validation of the "atomic switch" by Terabe et al. [8]. Additionally, by using switching time as a kinetic parameter that is, in contrast to the current, independent of the electronic properties, studies on pure ion conductors have been possible [70]. For resistance switching studies on transition metal oxides, STM has not been as widely used as CAFM. The main reason for this is the necessity of sufficient sample conductivity, not only in the low resistance state (LRS) but also in the high resistance state (HRS), to exclude surface charging effects. However, this issue can be avoided or minimised in many cases either by extrinsic doping [71, 72] or thermal reduction [73], enabling quantum mechanical tunnelling even on macroscopic insulators without significantly influencing ionic processes.

The first resistance switching phenomena induced by STM were found on ultrathin $\mathrm{SiO}_{2}$ and $\mathrm{Al}_{2} \mathrm{O}_{3}$ films. At the end of the last century Watanabe et al. observed local dielectric breakdown in $\mathrm{SiO}_{2}$ thin films by scanning the surface with a high negative tip potential. They attributed this to additional defect states formed by hot electron injection from the STM tip [74, 75]. Most switched 
areas showed metallic conductance after treatment, characterised as hard breakdown, whereas some smaller areas displayed non-metallic transport properties that were characterised as soft breakdown [75]. Magtoto et al. showed similar effects on an $\mathrm{Al}_{2} \mathrm{O}_{3}$ bilayer structure [76]. However, both effects were found to be irreversible. By carefully tuning the film thickness and the scanning parameters, Kurnosikov et al. were finally able to perform intentional reversible switching events, considered a precursor of soft breakdown, between different conductance states in $\mathrm{Al}_{2} \mathrm{O}_{3}$ [77].

The history of the study of resistance switching is strongly correlated with the investigation of high- $\kappa$ materials, which are also of great interest in charge-based memories such as DRAM. The ReRAM community also benefited from the great research effort in controlled growth of high- $\kappa$ materials. In particular, perovskites were of interest. Chen et al. investigated the resistance switching properties of $\mathrm{Nb}$-doped $\mathrm{SrTiO}_{3}$ in a combined STM/STS and XPS study [71]. By performing "write scans" using increased positive tip voltages $\geq 2 \mathrm{~V}$ they could show an increase of electron density in the following "read out scans" at lower voltages (corresponding to the LRS, see Figs. 20b and 20d). This observation was accompanied by an increase of the $\mathrm{Ti}^{3+}$ content in the sample and the creation of additional donor-like levels near the conduction band, detected in the $\mathrm{dI} / \mathrm{dV}-\mathrm{V}$ spectra. By using "write scans" with a negative tip voltage $\leq-2 \mathrm{~V}$ the electron density, and thus the surface conductivity, was significantly reduced (corresponding to the HRS, see Figs. 20a and 20c) showing the reversibility of the switching events. In the HRS both the $\mathrm{Ti}^{3+}$ signal and the state density of the donor-like levels became significantly lower (Fig. 20e). This supports the notion that oxygen vacancies have an important role in the resistance switching process of $\mathrm{SrTiO}_{3}$. Another similar example is that of a Pt-doped $\mathrm{Nb}_{2} \mathrm{O}_{5}$ RRAM device [72]. Figs. 20f-I shows the state of the pristine device; by increasing the tip voltage from $1 \mathrm{~V}$ to $3 \mathrm{~V}$ the number of conductive spots increases, indicating a SET process (Fig. 20f-II to Fig. 20f-IV). By inverting the tip voltage to -3 V, conductive spots disappear due to a RESET process (Fig. 20f-V). 

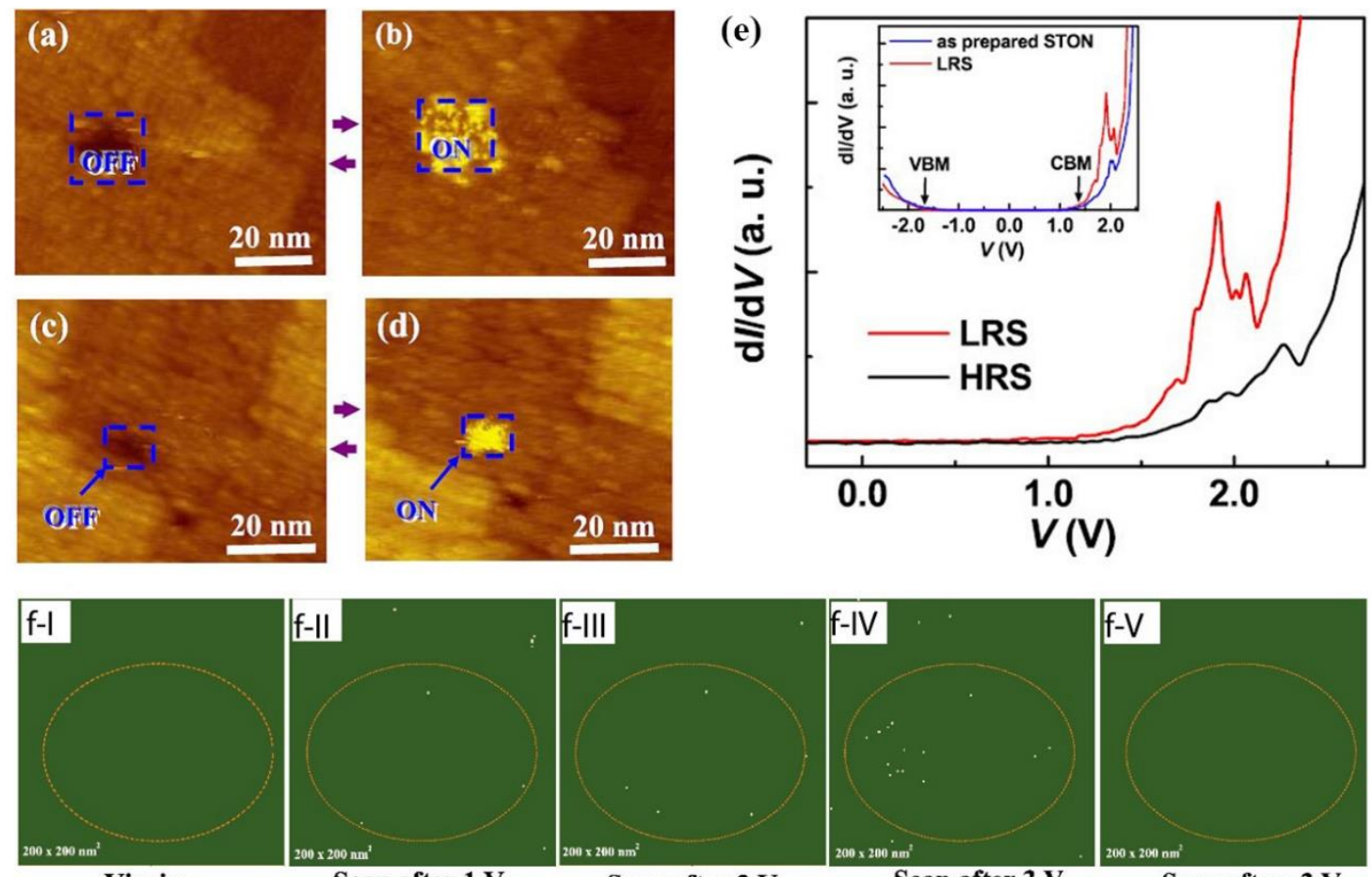

Scan after $1 \mathrm{~V}$

Scan after $2 \mathrm{~V}$

Scan after 3

Scan after $\mathbf{- 3} \mathrm{V}$

Fig. $20 \mathrm{STM}$ images of $\mathrm{Nb}$-doped $\mathrm{SrTiO}_{3}(\mathrm{STON}$ ) after scanning the marked area with positive tip voltages $\geq 2 \mathrm{~V}$ (a and b) and with negative tip voltages $\leq-2 \mathrm{~V}$ (c and d) and a tunnelling current set point of $0.5 \mathrm{nA}$; e) dI/dV-V curves obtained at LRS (corresponding to the ON area) and HRS (corresponding to the OFF area). Reprinted from [71] with permission. For comparison, the dI/dV-V curves for the as-prepared STON and LRS are given in the inset. (f-I)-(f-V) Current mapping as obtained from STM measurements on Pt-doped $\mathrm{Nb}_{2} \mathrm{O}_{5}$ subject to different tip biases. Reprinted from [72] with permission

Hota et al. demonstrated local resistance switching on complete $\mathrm{Au} / \mathrm{HfO}_{2} / \mathrm{In}_{0.53} \mathrm{Ga}_{0.47} \mathrm{As}$ cells [78]. In this study, current imaging tunnelling spectroscopy (CITS) was used to show the formation of conductive filaments consisting of partially reduced hafnium oxide during the SET process of a ReRAM cell. In CITS, an I-V curve is recorded at each pixel of an STM image, resulting in a conductance map of the surface [79]. By applying an increased positive tip voltage the authors could show non-uniform distributed spikes in the current profile, representing locations where conducting filaments had formed (Fig. 21a). Applying the opposite tip polarity reverses this effect (Fig. 21b). 
(a)

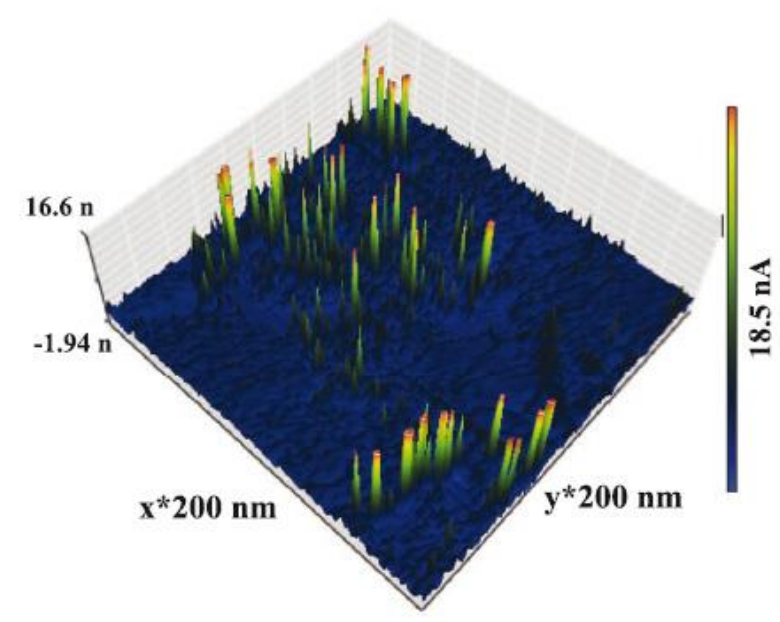

(b)

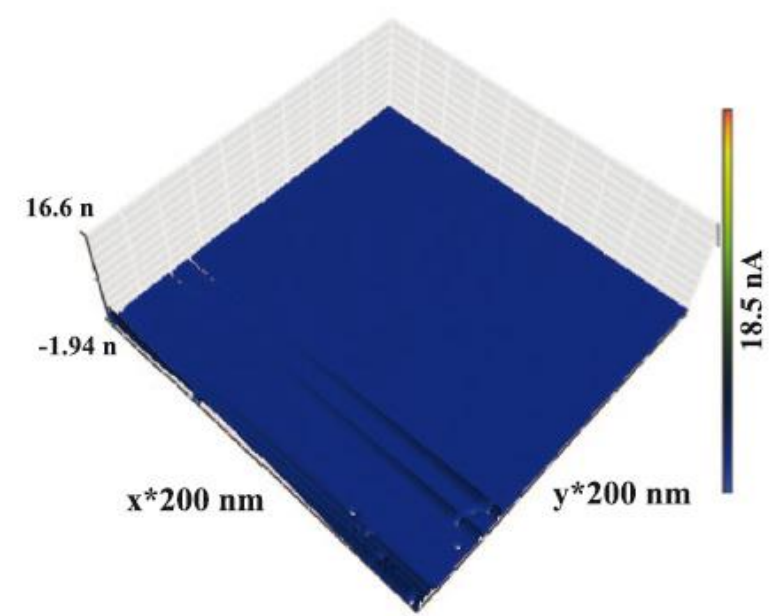

Fig. 21 CITS-STM images of an $\mathrm{Au} / \mathrm{HfO}_{2} / \mathrm{In}_{0.53} \mathrm{Ga}_{0.47} \mathrm{As}$ cell structure measured with a) positive and b) negative tip voltage. Reprinted from [78] with permission

The correlation between resistance changes induced by electrical stress and an increased defect concentration has been discussed since the study of resistance switching began, but the influence of the film morphology on the local distribution of these defects was not clear. STM measurements on thin $\mathrm{CeO}$ films indicated the significant role of grain boundaries [80]. These distinguished areas in polycrystalline systems have a strongly increased defect level. Thus they may behave as very active leakage paths in high- $\kappa$ gate dielectrics, as well as active switching centres in ReRAM cells. Mehonic et al. showed an enhanced conductivity at the edges of the columnar structures of sputter deposited silicon-rich $\mathrm{SiO}_{2}\left(\mathrm{SiO}_{\mathrm{x}}\right.$ where $\left.\mathrm{x} \approx 1.3\right)$ films [81]. These edges offer sites for the nucleation of oxygen vacancies and silicon nanoinclusions, which were found to be integral constituents of conductive filaments in $\mathrm{SiO}_{\mathrm{x}}$ resistance switches $[62,63]$. By placing the STM tip above a column edge and applying the appropriate voltage the sample could be reversibly switched between HRS and LRS [82]. As shown in Fig. 22a these conductive pathways around the columns are around 5-30 nm in diameter suggesting that silica based ReRAM cells may be scaled down to small nanometre-scale dimensions in order to achieve very high levels of integration. 
a)

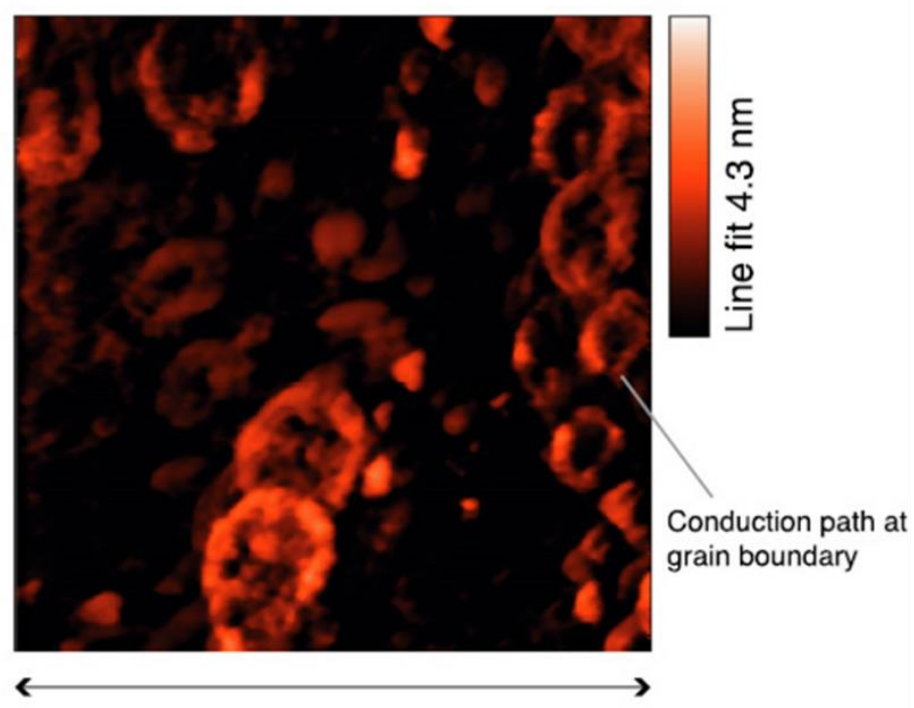

$340 \mathrm{~nm}$ b)
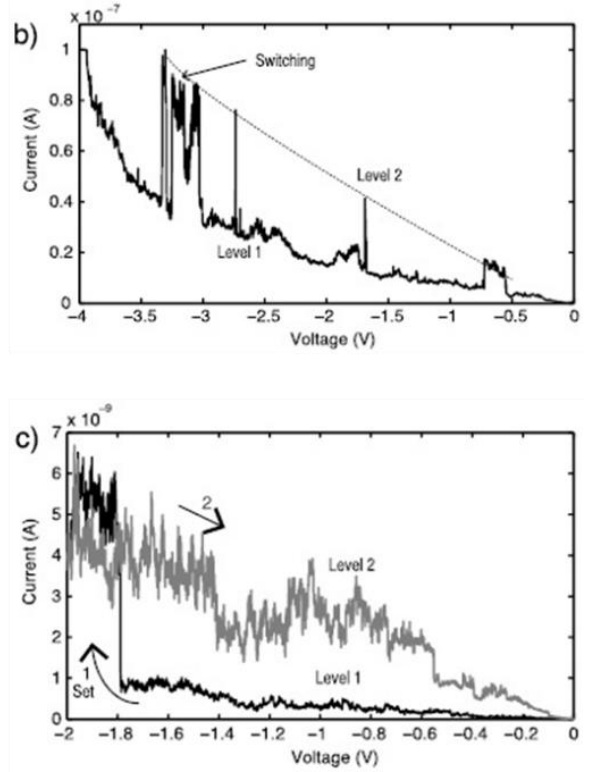

Fig. 22 (a) STM image of a sputter deposited $\mathrm{SiO}_{\mathrm{x}}$ film showing enhanced conductivity at column edges; (b) and (c) I-V curve obtained with STM spectroscopy showing resistance switching between two distinct states. Reprinted from [82] with permission

The high sensitivity of STM can also be used to study the switching properties of highly conducting oxide materials, whose properties are inaccessible with other techniques. Moors et al. demonstrated the resistance switching activity of $\mathrm{SrRuO}_{3}$ thin films [73]. Here, the switching area could be scaled down to approximately $2 \mathrm{~nm}$ in diameter. This strong electron conducting perovskite was generally viewed as an inactive switching component and was therefore used as a common electrode material in ReRAM cells.

In the same study, the authors also investigated the switching properties of the macroscopic insulator $\mathrm{Ta}_{2} \mathrm{O}_{5}$, demonstrating the applicability of STM to a wide spectrum of transition metal oxides [73]. Here, two different switching mechanisms were observed, depending on the stoichiometry of the samples. In the case of highly reduced $\mathrm{TaO}_{\mathrm{x}}$ films, the LRS was attained under a cathodic tip potential by reducing the $\mathrm{TaO}_{\mathrm{x}}$ locally to metallic $\mathrm{Ta}$, leading to an Ohmic $I-V$ characteristics (Fig. 23b). In contrast, reoxidised films exposed to ambient conditions showed a LRS under anodic tip potential by creating additional oxygen vacancies, resulting in non-Ohmic behaviour (see Fig. 23d). 

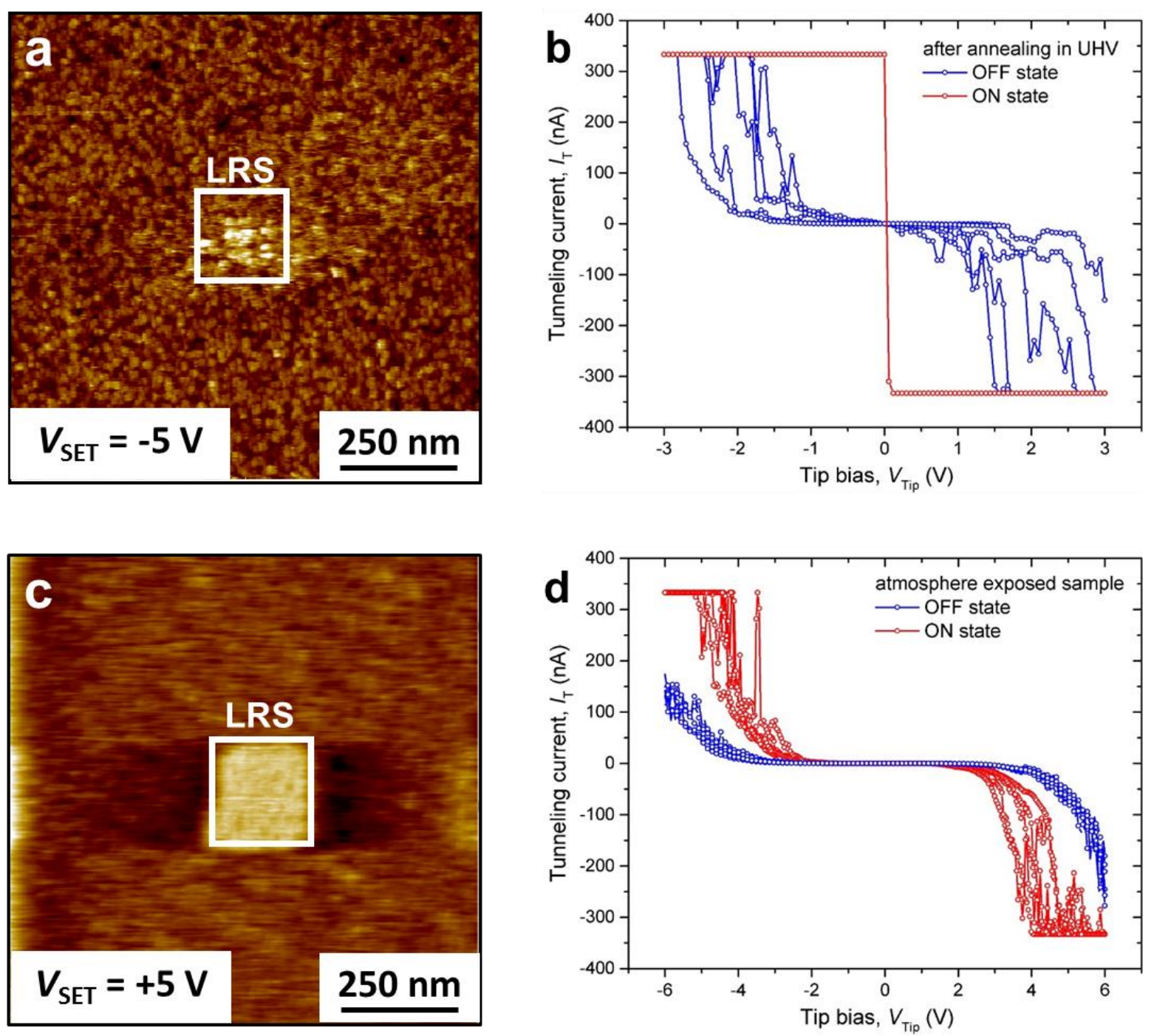

Fig. 23 (a) STM image of a vacuum annealed $\mathrm{TaO}_{\mathrm{x}}$ film after scanning the marked area with a cathodic tip voltage of -5 $\mathrm{V}$; (b) I-V curves measured on switched and unmodified regions of the strongly reduced sample; (c) STM image of an atmosphere exposed $\mathrm{TaO}_{\mathrm{x}}$ film after scanning the marked area with an anodic tip voltage of $+5 \mathrm{~V}$; (d) I-V curves measured on switched and unmodified regions of the post-oxidised sample. Reprinted from [73] with permission

Along with STM based resistance switching studies on classical oxide materials, more advanced material systems have also been investigated. Plecenik et al. studied the I-V characteristics of epitaxially grown c-axis oriented $\mathrm{YBa}_{2} \mathrm{Cu}_{3} \mathrm{O}_{7-\mathrm{x}}$ thin films [83]. This is a material known to be a high temperature superconductor [84]. Using a Pt-Ir STM tip as a top electrode, the authors observed a temperature-dependent resistance switching effect. Here, only the resistance of the HRS was increased significantly with decreasing temperature; the LRS was unaffected. At a fixed temperature, the resistance of the LRS decreased when the maximal current or voltage value was increased, while the resistance of the HRS remained stable. Dubost et al. investigated a new form of resistance 
switching within the Mott insulator compound $\mathrm{GaTa}_{4} \mathrm{Se}_{8}$ [85]. By applying electric pulses with an STM tip to the sample, an electronic phase separation concomitant with a lattice deformation could be generated. In the transited state both metallic and strongly insulating domains with a size ranging between 30 and $50 \mathrm{~nm}$ coexisted within the semiconducting material.

Finally, in order to reach the maximum scalability of resistance switching devices, the electrical characterisation of single nanoparticles became a focus of the ReRAM community. Here, the high resolution of STM and its ability to precisely contact nanoscale objects was crucial. Gambardella et al. investigated $\mathrm{Co}$ nanoparticles embedded in a $\mathrm{TiO}_{2}$ matrix and showed local resistance switching in the tunnel conductivity at the level of a single nanoparticle by applying voltage pulses through the STM tip [86]. Hota et al. integrated $\mathrm{Au}$ nanoparticles in an $\mathrm{Nb}_{2} \mathrm{O}_{5}$ matrix and studied their influence on the conductive filament formation and rupture during the SET/RESET process [87]. STM measurements using the CITS technique evidenced an enhanced filament formation and rupture in the presence of nanodots. The authors considered that the large effective surface of the Au/oxide interface resulted in an increased oxygen ion storage at the nanodot locations. Chakrabarti et al. used a copper-zinc-tin-sulphide shell to encapsulate $\mathrm{Au}$ nanoparticles [88]. These complex particles showed two transitions in the tunnelling conductance and, hence, multilevel switching following the application of different voltage pulses with the STM tip. Another promising approach is the use of carbon nanotubes as switching elements due to their relatively simple integration in silicon technology [89]. Ageev et al. showed that vertically aligned carbon nanotubes grown on a metallic substrate by plasma enhanced chemical vapour deposition could switch between two conductance states with different voltage pulses [90]. The proposed switching mechanism related to the internal electric field strength of a nanotube caused by a voltage pulse induced instantaneous deformation [91].

\section{Concluding remarks}

In summary, we have given an overview of the in situ TEM and STM characterizations that have been performed to disclose the localized electrochemical processes in different types of resistance switching devices. As a high resolution technique with versatile and powerful analytical 
capabilities, in situ TEM studies employing carefully-designed sample structures have not only visualized the formation of metal filaments and identified their static properties, but also clarified detailed filament dynamics, including the growth directions and geometries. These findings give rise to a generalized picture for filament growth, in which the different growth modes can be explained by detailed kinetic parameters including ion mobility and redox rate. The resistance switching in oxide-based ReRAM is manifested as formation and rupture of oxygen-deficient conduction channels, e.g. filaments in the $\mathrm{TiO}_{\mathrm{x}}$ Magnéli phase, driven by an applied electric field and/or thermal effects. Furthermore, TEM imaging during cyclic operations has revealed the dependence of filament characteristics on compliance currents and nucleation positions, and thus offered implications for device reliabilities. In contrast to the conventional CAFM method, the adoption of STM in resistance switching characterization has the advantages of eliminating tip-sample contact-induced deformations and greatly improved spatial resolution, and have been used to understand the resistance switching mechanisms in a wide spectrum of materials ranging from transition metal oxides, highly conductive oxides and superconductors to Mott insulators, nanoparticles and carbon nanotubes. Thanks to these microscopic studies, our understanding of the switching mechanisms of ReRAM have been significantly improved recently, which will in turn facilitate the modelling and optimization of the devices.

Despite the encouraging progress in understanding the switching mechanisms, especially that of CBRAM devices, continued research efforts are required to further clarify the detailed switching mechanisms- particularly in the case VCM devices, which are more difficult to study. In addition, it will be of paramount significance to establish correlations between structural evolution at the microscopic level and the variation, degradation and failure of resistance switching devices, which can be used to guide future device optimization. Looking forward, TEM and STM techniquess will undoubtedly continue to play important roles in disclosing the detailed physicochemical mechanisms of resistance switching systems, and the findings from these studies may be of general significance for device engineering, physical chemistry, materials science, and nanotechnology.

\section{Acknowledgements}


Y.Y. acknowledges financial support from National Science Foundation of China (61674006, 61421005, 61376087 and 61574007), Beijing Municipal Science \& Technology Commission Program (Z161100000216148) as well as the "1000 Youth Talents Program” of China and also thanks Mr. Jingxian Li for his assistance. M.B., A.M. and A.J.K. gratefully acknowledge financial support from the Engineering and Physical Sciences Research Council (EPSRC). Y.T., A.T.F. and M.A. acknowledge financial support from the Japan Society for the Promotion of Science (JSPS, KAKENHI, 15H01706, 16H0433906 and 16K18073). Y.Y. prepared sections 1-2. Y.T., A.T.F. and M.A. prepared sections 3-4. M.M., M.B., A.M. and A.J.K. prepared section 5. All authors contributed to section 6, revised and discussed the whole manuscript at all stages. 


\section{References}

1. L. O. Chua, IEEE Trans. Circuit Theory 18, 507 (1971).

2. R. Waser, M. Aono, Nat. Mater. 6, 833 (2007).

3. H.-S. P. Wong, H.-Y. Lee, S. Yu, Y.-S. Chen, Y. Wu, P.-S. Chen, B. Lee, F. T. Chen, M.-J. Tsai, Proc. IEEE 100, 1951 (2012).

4. J. Borghetti, G. S. Snider, P. J. Kuekes, J. J. Yang, D. R. Stewart, R. S. Williams, Nature 464, 873 (2010).

5. S. H. Jo, T. Chang, I. Ebong, B. B. Bhadviya, P. Mazumder, W. Lu, Nano Lett. 10, 1297 (2010).

6. I. Valov, W. D. Lu, Nanoscale 8, 13828 (2016).

7. K. Sozt, W. Speier, G. Bihlmayer, R. Waser, Nat. Mater. 5, 312 (2006).

8. K. Terabe, T. Hasegawa, T. Nakayama, M. Aono, Nature 433, 47 (2005).

9. Y. Yang, P. Gao, S. Gaba, T. Chang, X. Pan, W. Lu, Nat. Commun. 3, 732 (2012).

10. Y. Yang, P. Gao, L. Li, X. Pan, S. Tappertzhofen, S. Choi, R. Waser, I. Valov, W. D. Lu, Nat. Commun. 5, 4232 (2014).

11. D. H. Kwon, K. M. Kim, J. H. Jang, J. M. Jeon, M. H. Lee, G. H. Kim, X. S. Li, G. S. Park, B. Lee, S. Han, M. Kim, C. S. Hwang, Nat. Nanotechnol. 5, 148 (2010).

12. M. Arita, A. Takahashi, Y. Ohno, A. Nakane, A. Tsurumaki-Fukuchi, Y. Takahashi, Sci. Rep. 5, 17103 (2015).

13. Q. Liu, J. Sun, H. Lv, S. Long, K. Yin, N. Wan, Y. Li, L. Sun, M. Liu, Adv. Mater. 24, 1844 (2012).

14. X. Z. Tian, S. Z. Yang, M. Zeng, L. F. Wang, J. K. Wei, Z. Xu, W. L. Wang, X. D. Bai, Adv. Mater. 26, 3649 (2014).

15. W. A. Hubbard, A. Kerelsky, G. Jasmin, E. R. White, J. Lodico, M. Mecklenburg, B. C. Regan, Nano Lett. 15, 3983 (2015).

16. Y. Yang, W. D. Lu, IEEE Trans. Nanotechnol. 15, 465 (2016).

17. T. Fujii, M. Arita, Y. Takahashi, I. Fujiwara, Appl. Phys. Lett. 98, 212104 (2011).

18. Z. Xu, Y. Bando, W. L. Wang, X. D. Bai, D. Golberg, ACS Nano 4, 2515 (2010).

19. L. Chen, Z. G. Liu, Y. D. Xia, K. B. Yin, L. G. Gao, J. Yin, Appl. Phys. Lett. 94, 162112 (2009).

20. S.-J. Choi, G.-S. Park, K.-H. Kim, S. Cho, W.-Y. Yang, X.-S. Li, J.-H. Moon, K.-J. Lee, K. Kim, 
Adv. Mater. 23, 3272 (2011).

21. J. Sun, Q. Liu, H. W. Xie, X. Wu, F. Xu, T. Xu, S. B. Long, H. B. Lv, Y. T. Li, L. T. Sun, M. Liu, Appl. Phys. Lett. 102, 053502 (2013).

22. T. Sakamoto, K. Lister, N. Banno, T. Hasegawa, K. Terabe, M. Aono, Appl. Phys. Lett., 91, 092110 (2007).

23. Y. C. Yang, F. Pan, Q. Liu, M. Liu, F. Zeng, Nano Lett. 9, 1636 (2009).

24. X. Guo, C. Schindler, S. Menzel, R. Waser, Appl. Phys. Lett. 91, 133513 (2007).

25. K. Krishnan, T. Tsuruoka, C. Mannequin, M. Aono, Adv. Mater. 28, 640 (2016).

26. C. P. Hsiung, H. W. Liao, J. Y. Gan, T. B. Wu, J. C. Hwang, F. Chen, M. J. Tsai, ACS Nano 4, $5414(2010)$.

27. Z. Wang, H. Jiang, M. Hyung Jang, P. Lin, A. Ribbe, Q. Xia, J. J. Yang, Nanoscale 8, 14023 (2016).

28. S. Gao, C. Song, C. Chen, F. Zeng, F. Pan, Appl. Phys. Lett. 102, 141606 (2013).

29. I. Valov, R. Waser, J. R. Jameson, M. N. Kozicki, Nanotechnology 22, 254003 (2011).

30. T. Fujii, M. Arita, Y. Takahashi, I. Fujiwara, J. Mater. Res. 27, 886 (2012).

31. M. Arita, Y. Ohno, Y. Murakami, K. Takamizawa, A. Tsurumaki-Fukuchi, Y. Takahashi, Nanoscale 8, 14754 (2016).

32. M. Kudo, Y. Ohno, K. Hamada, M. Arita, Y. Takahashi, ECS Trans. 58, 19 (2013).

33. M. Kudo, M. Arita, Y. Ohno, Y. Takahashi, Appl. Phys. Lett. 105, 173504 (2014).

34. M. Arita, Y. Ohno, Y. Takahashi, Phys. Status Solidi A 213, 306 (2016).

35. M. Kudo, M. Arita, Y. Ohno, T. Fujii, K. Hamada, Y. Takahashi, Thin Solid Films 533, 48 (2013).

36. N. Banno, T. Sakamoto, N. Iguchi, H. Sunamura, K. Terabe, T. Hasegawa, M. Aono, IEEE Trans. Electron Devices 55, 3283 (2008).

37. R. Waser, R. Dittmann, G. Staikov, K. Szot, Adv. Mater. 21, 2632 (2009).

38. L. A. Escobar, W. Q. Meeker, Statist. Sci. 21, 552 (2006).

39. M. Kudo, M. Arita, Y. Takahashi, K. Ohba, M. Shimuta, I. Fujiwara, Proc. 7th Internat. Memory Workshop (IMW), IEEE, Piscataway, 85 (2015). DOI: 10.1109/IMW.2015. 7150312.

40. J. Zahurak, K. Miyata, M. Fischer, M. Balakrishnan, S. Chhajed, D. Wells, H. Li, A. Torsi, J. Lim, M. Korber, K. Nakazawa, S. Mayuzumi, M. Honda, S. Sills, S. Yasuda, A. Calderoni, B. 
Cook, G. Damarla, H. Tran, B. Wang, C. Cardon, K. Karda, J. Okuno, A. Johnson, T. Kunihiro, J. Sumino, M. Tsukamoto, K. Aratani, N. Ramaswamy, W. Otsuka, K. Prall, IEEE Int. Electron Devices Meet. 6.2.1/140 (2014).

41. Y. Takahashi, M. Kudo, M. Arita, ECS Trans. 69, 299 (2015).

42. A. Sawa, Mater. Today 11, 28 (2008).

43. P. R. Mickel, A. J. Lohn, M. J. Marinella, Mod. Phys. Lett. B 28, 1430003 (2014).

44. A. Mehonic, M. Buckwell, L. Montesi, M. S. Munde, D. Gao, S. Hudziak, R. J. Chater, S. Fearn, D. McPhail, M. Bosman, A. L. Shluger, A. J. Kenyon, Adv. Mater. 28, 7486 (2016).

45. H. Schroeder, R. Pandian, J. Miao, Phys. Status Solidi A 208, 300 (2011).

46. T. Fujii, M. Arita, K. Hamada, H. Kondo, H. Kaji, Y. Takahashi, M. Moniwa, I. Fujiwara, T. Yamaguchi, M. Aoki, Y. Maeno, T. Kobayashi, M. Yoshimaru, J. Appl. Phys. 109, 053702 (2011).

47. T. Fujii, M. Arita, K. Hamada, Y. Takahashi, N. Sakaguchi, J. Appl. Phys. 113, 083701 (2013).

48. Z. Fan, X. D. Fan, A. Li, L. X. Dong, Nanoscale 5, 12310 (2013).

49. J. P. Strachan, M. D. Pickett, J. J. Yang, S. Aloni, A. L. D. Kilcoyne, G. Medeiros-Ribeiro, R. S. Williams, Adv. Mater. 22, 3573 (2010).

50. K. M. Kim, S. J. Song, G. H. Kim, J. Y. Seok, M. H. Lee, J. H. Yoon, J. Park, C. S. Hwang, Adv. Funct. Mater. 21, 1587 (2011).

51. J. Y. Chen, C. L. Hsin, C. W. Huang, C. H. Chiu, Y. T. Huang, S. J. Lin, W. W. Wu, L. J. Chen, Nano Lett. 13, 3671 (2013).

52. G.-S. Park, Y. B. Kim, S. Y. Park, X. S. Li, S. Heo, M.-J. Lee, M. Chang, J. H. Kwon, M. Kim, U. I. Chung, R. Dittmann, R. Waser, K. Kim, Nat. Commun. 4, 2382 (2013).

53. X. Wu, D. Cha, M. Bosman, N. Raghavan, D. B. Migas, V. E. Borisenko, X.-X. Zhang, K. Li, K.-L. Pey, J. Appl. Phys. 113, 114503 (2013).

54. Z. Wei, T. Takagi, Y. Kanzawa, Y. Katoh, T. Ninomiya, K. Kawai, S. Muraoka, S. Mitani, K. Katayama, S. Fujii, R. Miyanaga, Y. Kawashima, T. Mikawa, K. Shimakawa, K. Aono, IEEE Int. Electron Devices Meet. 31.4.1/721 (2011).

55. C. Li, Y. Yao, X. Shen, Y. G. Wang, J. J. Li, C. Z. Gu, R. C. Yu, Q. Liu, M. Liu, Nano Res. 8, $3571(2015)$.

56. T. Fujii, H. Kaji, H. Kondo, K. Hamada, M. Arita, Y. Takahashi, IOP Conf. Ser.: Mater. Sci. Eng. 
8, 012033 (2010).

57. Y. Yang, W. Lü, Y. Yao, J. Sun, C. Gu, L. Gu, Y. Wang, X. Duan, R. Yu, Sci. Rep. 4, 3890 (2014).

58. J. Norpoth, S. Mildner, M. Scherff, J. Hoffmann, C. Jooss, Nanoscale 6, 9852 (2014).

59. C. Jooss, J. Hoffmann, J. Fladerer, M. Ehrhardt, T. Beetz, L. Wu, Y. Zhu, Phys. Rev. B 77, 132409 (2008).

60. Z. Liao, P. Gao, X. Bai, D. Chen, J. Zhang, J. Appl. Phys. 111, 114506 (2012).

61. P. Gao, Z. Z. Wang, W. Y. Fu, Z. L. Liao, K. H. Liu, W. L. Wang, X. D. Bai, E. Wang, Micron 41, $301(2010)$.

62. J. Kwon, M. Skowronski, A. A. Sharma, J. A. Bain, IEEE Internat. Reliability Phys. Symp. 5E.5 (2014). DOI:10.1109/IRPS.2014. 6860680.

63. M. Buckwell, L. Montesi, S. Hudziak, A. Mehonic, A. J. Kenyon, Nanoscale 7, 18030 (2015).

64. M. P. Murrell, M. E. Welland, S. J. O’Shea, T. M. H. Wong, J. R. Barnes, A. W. McKinnon, M. Heyns, S. Verhaverbeke, Appl. Phys. Lett. 62, 786 (1993).

65. M. Lanza, Materials 7, 2155 (2014).

66. U. Celano, L. Goux, A. Belmonte, K. Opsomer, A. Franquet, A. Schulze, C. Detavernier, O. Richard, H. Bender, M. Jurczak, W. Vandervorst, Nano Lett. 14, 2401 (2014).

67. J. Hou, B. Rouxel, W. Qin, S. Nonnenmann, D. A. Bonnell, Nanotechnol. 24, 395703 (2013).

68. B. Singh, D. Varandani, B. R. Mehta, Appl. Phys. Lett. 103, 051604 (2013).

69. A. Wedig, M. Luebben, D. -Y. Cho, M. Moors, K. Skaja, V. Rana, T. Hasegawa, K. K. Adepalli, B. Yildiz, R. Waser, I. Valov, Nat. Nanotechnol. 11, 67 (2016).

70. I. Valov, I. Sapezanskaia, A. Nayak, T. Tsuruoka, T. Bredow, T. Hasegawa, G. Staikov, M. Aono, R. Waser, Nat. Mater. 11, 530 (2012).

71. Y. L. Chen, J. Wang, C. M. Xiong, R. F. Dou, J. Y. Yang, J. C. Nie, J. Appl. Phys. 112, 023703 (2012).

72. M. K. Hota, M. K. Bera, S. Verma, C. K. Maiti, Thin Solid Films 520, 6648 (2012).

73. M. Moors, K. K. Adepalli, Q. Lu, A. Wedig, C. Bäumer, K. Skaja, B. Arndt, H. L. Tuller, R. Dittmann, R. Waser, B. Yildiz, I. Valov, ACS Nano 10, 1481 (2016).

74. H. Watanabe, K. Fujita, M. Ichikawa, Appl. Phys. Lett. 72, 1987 (1998).

75. H. Watanabe, T. Baba, M. Ichikawa, J. Appl. Phys. 85, 6704 (1999).

76. N. P. Magtoto, C. Niu, B. M. Ekstrom, S. Addepalli, J. A. Kelber, Appl. Phys. Lett. 77, 2228 
(2000).

77. O. Kurnosikov, F. C. de Nooij, P. LeClair, J. T. Kohlhepp, B. Koopmans, H. J. M. Swagten, W. J. M. de Jonge, Phys. Rev. B 64, 153407 (2001).

78. M. K. Hota, C. Mukherjee, T. Das, C. K. Maiti, ECS J. Solid State Sci. Technol. 1, N149 (2012).

79. R. J. Hamers, R. M. Tromp, J. E. Demuth, Phys. Rev. Lett. 56, 1972 (1986).

80. K. Shubhakar, K. L. Pey, S. S. Kushvaha, S. J. O’Shea, N. Raghavan, M. Bosman, M. Kouda, K. Kakushima, H. Iwai, Appl. Phys. Lett. 98, 072902 (2011).

81. A. Mehonic, S. Cueff, M. Wojdak, S. Hudziak, O. Jambois, C. Labbé, B. Garrido, R. Rizk, A. J. Kenyon, J. Appl. Phys. 111, 074507 (2012).

82. A. Mehonic, S. Cueff, M. Wojdak, S. Hudziak, C. Labbé, R. Rizk, A.J. Kenyon, Nanotechnol. 23, 455201 (2012).

83. A. Plecenik, M. Tomasek, T. Plecenik, M. Truchly, J. Noskovic, M. Zahoran, T. Roch, M. Belogolovskii, M. Spankova, S. Chromik, P. Kus, Appl. Surf. Sci. 256, 5684 (2010).

84. A. Narlikar, Studies of High Temperature Superconductors, Nova Sci. Publ., New York, 1996.

85. V. Dubost, T. Cren, C. Vaju, L. Cario, B. Corraze, E. Janod, F. Debontridder, D. Roditchev, Nano Lett. 13, 3648 (2013).

86. A. Gambardella, M. Prezioso, M. Cavallini, Sci. Rep. 4, 4196 (2014).

87. M. K. Hota, M. K. Bera, C. K. Maiti, Nanosci. Nanotechnol. Lett. 4, 394 (2012).

88. S. Chakrabarti, A. J. Pal, Nanoscale 7, 9886 (2015).

89. M. Meyyappan, J. Phys. D: Appl. Phys. 42, 213001 (2009).

90. O. A. Ageev, Y. F. Blinov, O. I. Il'in, A. S. Kolomiitsev, B. G. Konoplev, M. V. Rubashkina, V. A. Smirnov, A. A. Fedotov, Tech. Phys. 58, 1831 (2013).

91. O. A. Ageev, Y. F. Blinov, O. I. Il'in, B. G. Konoplev, M. V. Rubashkina, V. A. Smirnov, A. A. Fedotov, Phys. Solid State 57, 825 (2015). 